OPEN ACCESS

Edited by:

Hiroaki Suetake,

Fukui Prefectural University, Japan

Reviewed by:

Kang-le Lu,

Jimei University, China

Patricia Diaz-Rosales,

Centro de Investigación en Sanidad

Animal (CISA), Spain

*Correspondence:

Wenbing Zhang

wzhang@ouc.edu.cn

Specialty section: This article was submitted to Comparative Immunology, a section of the journal

Frontiers in Immunology

Received: 15 March 2021 Accepted: 17 June 2021

Published: 01 July 2021

Citation:

Pan M, Liu J, Huang D, Guo Y, Luo K, Yang $M$, Gao $W, X u Q$, Zhang $W$ and Mai K (2021) FoxO3 Modulates LPS-

Activated Hepatic Inflammation in

Turbot (Scophthalmus maximus L.).

Front. Immunol. 12:679704.

doi: 10.3389/fimmu.2021.679704

\section{FoxO3 Modulates LPS-Activated Hepatic Inflammation in Turbot (Scophthalmus maximus L.)}

\author{
Mingzhu Pan ${ }^{1}$, Jiahuan Liu ${ }^{1}$, Dong Huang ${ }^{1}$, Yanlin Guo ${ }^{1}$, Kai Luo ${ }^{1}$, Mengxi Yang ${ }^{1}$, \\ Weihua $\mathrm{Gao}^{2}$, Qiaoqing $\mathrm{Xu}^{2}$, Wenbing Zhang ${ }^{1,2^{\star}}$ and Kangsen $\mathrm{Mai}^{1,2}$ \\ 1 The Key Laboratory of Mariculture (Ministry of Education), The Key Laboratory of Aquaculture Nutrition and Feeds (Ministry \\ of Agriculture and Rural Affairs), Fisheries College, Ocean University of China, Qingdao, China, ${ }^{2}$ Department of Fisheries, \\ College of Animal Science, Yangtze University, Jingzhou, China
}

In mammals, forkhead box $\mathrm{O} 3$ (foxo3) plays important roles in liver immune system. The foxo3 can regulate cell cycle, DNA repair, hypoxia, apoptosis and so on. However, as such an important transcription factor, few studies on foxo3 in fish have been reported. The present study characterized the foxo3 in turbot (Scophthalmus maximus L.). Lipopolysaccharide (LPS) incubated in vitro (hepatocytes) and injected in vivo (turbot liver) were used to construct inflammatory models. The foxo3 was interfered and overexpressed to investigate its functions in liver inflammation. The open reading frame (ORF) of foxo3 was 1998 bp (base pair), encoding 665 amino acids. Sequence analysis showed that foxo3 of turbot was highly homologous to other fishes. Tissue distribution analysis revealed that the highest expression of foxo 3 was in muscle. Immunofluorescence result showed that foxo3 was expressed in cytoplasm and nucleus. Knockdown of foxo3 significantly increased mRNA levels of tumor necrosis factor- $\alpha$ (tnf- $\alpha$ ), interleukin-1 $\beta$ (il-1 $\beta$ ), interleukin-6 (il-6), myeloid-differentiation factor 88 (myd88), cd83, toll-like receptor 2 (t/r-2) and protein level of c-Jun N-terminal kinase (JNK) in sifoxo3 + LPS (siRNA of foxo3+ LPS) group compared with NC + LPS (negative control + LPS) group in turbot hepatocytes. Overexpressed foxo3 significantly decreased mRNA levels of thf- $\alpha$, il-6, nuclear transcription factor-kappa B (nf- $k b), c d 83$, thr-2 and the protein level of JNK in vitro. In vivo analysis, foxo3 knockdown significantly increased levels of GOT in serum after LPS injection compared with NC+LPS group. Overexpressed foxo3 significantly decreased levels of GPT and GOT in pcDNA3.1-foxo3+LPS group compared with pcDNA3.1+LPS group in vivo. Foxo3 knockdown significantly increased mRNA levels of tnf- $\alpha$, il-1 $\beta$, il-6, nf- $\kappa b$, myd88 and protein level of JNK in vivo in sifoxo3 +LPS group compared with NC+LPS group in turbot liver. Overexpressed foxo3 significantly decreased mRNA levels of il-1 $\beta$, il-6, myd88, cd83, jnk and protein level of JNK in pcDNA3.1-foxo3+LPS group compared with pcDNA3.1+LPS group in turbot liver. The results indicated that foxo3 might modulate LPS-activated hepatic inflammation in turbot by decreasing the proinflammatory cytokines, the levels of GOT and GPT as well as activating JNK/caspase-3 and t/r-2/myd88/nf- $\kappa$ b pathways. Taken together, these findings indicated that $\mathrm{FoxO} 3$ may play important roles in liver immune responses to 


\section{LPS in turbot and the research of FoxO3 in liver immunity enriches the studies on immune regulation, and provides theoretical basis and molecular targets for solving liver inflammation and liver injury in fish.}

Keywords: turbot, forkhead box 03 , inflammation, immune, lipopolysaccharide

\section{INTRODUCTION}

The forkhead $\mathrm{O}$ (FoxO) family has a highly conserved amino acid sequence called the forkhead domain (FD). Transcription factors of FoxO subfamily include FoxO1, FoxO3, FoxO4 and FoxO6 in mammals. Members of the FoxO family regulate a variety of cellular functions, such as cell differentiation, metabolism, proliferation and apoptosis $(1,2)$. Many studies have shown that FoxO is an essential regulator of different liver diseases (3). Mice that are deficient in FoxO1/3/4 specifically in hepatocytes are sensitive to high-fat cholesterol diet-induced inflammation and liver injury (4). FoxO1/3/4 in hepatocytes play a protective role in diet-induced liver fibrosis. The role of FoxOs in the modulation of immune response and inflammation is essential (5). In the studies of mice, lipopolysaccharide (LPS) administration decreased FoxO activity and increased nuclear transcription factor-kappa B (NF- $\mathrm{\kappa B}$ ) activity (6). In general, the FoxO family plays a crucial role in immunity.

FoxO3 is one of the most important members of the FoxO family. In mammals, FoxO3 has been found to be a multifunctional transcription factor involved in regulating gene expression in the cell cycle, DNA repair, hypoxia and apoptosis (7-12). In mice, overexpression of FoxO3 improves inflammatory status in by decreasing the levels $i l-1 \beta$, il-6, caspase- 3 and other genes (13). Interleukin-1 $\beta$ (il-1 $\beta$ ), interleukin-6 (il-6) and tumor necrosis factor- $\alpha(t n f-\alpha)$ are pro-inflammatory cytokines that are the major markers of inflammation (14). The liver is a main metabolic and alexipharmic organ and is vital for host defense and tissue repair in infection (15). In the study of mice liver, it was reported that overexpression of $\mathrm{FoxO} 3$ attenuated liver failure by downregulating the expressions of $\operatorname{tnf}-\alpha, i l-1 \beta$ and il-6 (16). In addition, it was demonstrated that $\mathrm{FoxO} 3$ protected the liver from ethanol-induced inflammation in mice (17). Based on the data above, FoxO3 plays a vital role in liver immune regulation.

Although many studies on FoxO3 have been carried out in mammals, research of FoxO3 in fish is still severely lacking. It was found that the mitochondrial autophagy pathway in liver of large yellow croaker (Larimichthys crocea) was regulated by the FoxO3 (18). Dietary L-leucine attenuated the protein degradation via regulating the protein kinase $\mathrm{B}$ (AKT)/FoxO3 signaling pathway in hybrid catfish (Pelteobagrus vachelli $\times$ Leiocassis longirostris) (19). In addition, it was reported that the expression foxo3 was significantly upregulated in channel catfish (Ictalurus punctatus) after bacterial infection (20). Hence, it is necessary to investigate the molecular immune mechanism of foxo3 in fish.

Turbot (Scophthalmucs maximus L.) is one of the most commercially important marine fish species, which was widely cultured in Europe and Asia (21). Turbot culture represents a large proportion of land-based tank-cultured fish in China, especially in recirculating aquaculture systems. What's more, turbot industries worldwide have suffered heavily from disease outbreaks caused by bacterial infections including Edwardsiella tarda and different species of Vibrio, such as V. anguillarum (2224). These caused huge economic losses to the turbot industry. Therefore, it is indispensable to study the molecular immune mechanism of turbot. There were few studies on foxo 3 in turbot, and the only report was that foxo 3 belongs to chemokine signaling pathway enriched in omics study of turbot (25). The role of foxo 3 in the immune of turbot is unclear. LPS is found in the outer membrane of Gram-negative bacteria. Recent evidences from different animal models indicate that LPSinduced acute liver injury (26). Therefore, LPS incubated in vitro and injected in vivo were used to construct liver inflammatory models to determine whether foxo 3 regulates immune signaling pathway in turbot liver. It helps to improve the understanding of the functions of foxo 3 in turbot even in carnivorous fish.

\section{MATERIALS AND METHODS}

The protocols for animal care and handing used in this study were approved by the Institutional Animal Care and Use Committee of Ocean University of China.

\section{Animals}

Turbots (Scophthalmus maximus L.) (10.0 $\pm 0.5 \mathrm{~g})$ were purchased from a commercial farm (Yantai, Shandong Province, China) and maintained at $20 \pm 1^{\circ} \mathrm{C}$ in aerated seawater. The fish were fed with a commercial feed (crude protein: $48 \%$; crude lipid: $10 \%$ ) twice daily and acclimated to the laboratory setting for two weeks before all the experiments.

\section{Molecular Cloning of Turbot Foxo3 and Sequence Analysis}

The cloning of turbot foxo 3 was performed by slightly modifying the previously described methods (21). Total mRNA was extracted from turbot liver using Trizol Reagent (Invitrogen, USA). The quality and concentration of RNA were measured by Nanodrop 2000 (Thermo Fisher Scientific, USA). Then, the quality-compliant RNA (1000 ng) was reversely transcribed into cDNA using the PrimeScript $^{\mathrm{TM}}$ RT reagent Kit with gDNA Eraser (RR047A, Takara, Japan), and amplification systems and procedures were performed according to the manufacturer's instructions. PCR primers for CDS-foxo3 cloning were designed as in Table 1. The PCR amplification system was performed with the following 
TABLE 1 | Sequences of the gPCR primers.

\begin{tabular}{|c|c|c|}
\hline Primers & Sequence $\left(5^{\prime}-3^{\prime}\right)$ & GenBank Accession Number \\
\hline CDS-foxo3-R & TCAGCCAGGTACCCTCAGCCAGGT & \\
\hline $\operatorname{tnf} \alpha-\mathrm{F}$ & GGGTGGATGTGGAAGGTGAT & FJ654645.1 \\
\hline $\operatorname{tnf} \alpha-\mathrm{R}$ & GGCCTCTGTTTGGCTTGACT & \\
\hline$i l-1 \beta-\mathrm{F}$ & GCGACATGGTGCGATTTCTG & AWP07584.1 \\
\hline il-6-R & TTGTGGTTGCTGGAGGG & \\
\hline$n f-\kappa b-F$ & ACACTGCTGAGCTGAAGATC & MF370855 \\
\hline$n f-\kappa b-R$ & CTCTGAGCCCATCAGGGTC & \\
\hline myd88-F & CCCAATGGTAGCCCTGAGAT & KP985236 \\
\hline myd88-R & CATCTCGGTCGAACACACAC & \\
\hline cd83-F & AGTACTACGTCGGCTTGGAC & HM159997.1 \\
\hline t/r2-R & CTCGTCGATGCTCCTCTGAT & \\
\hline$\beta$-actin-F & GTAGGTGATGAAGCCCAGAGCA & EU686692.1 \\
\hline$\beta$-actin-R & CTGGGTCATCTTCTCCCTGT & \\
\hline
\end{tabular}

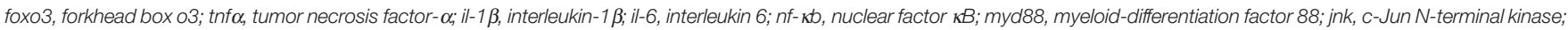
trl-2, toll-like receptor 2.

parameters: initial denaturation at $95^{\circ} \mathrm{C}$ for $2 \mathrm{~min}$ and 35 cycles of $95^{\circ} \mathrm{C}$ for $5 \mathrm{~s}, 55^{\circ} \mathrm{C}$ for $30 \mathrm{~s}$ and $72^{\circ} \mathrm{C}$ for $1 \mathrm{~min}$, followed by $72^{\circ} \mathrm{C}$ for $10 \mathrm{~min}$. The PCR product was analyzed in $1.2 \%$ agarose gel electrophoresis, purified, and ligated to the pEASY-Blunt cloning vector (CB101-01, TransGen, China). Then, the vector was transformed into Trans1-T1 Phage Resistant Chemically Competent Cell (CD501-02, TransGen, China). The PCR products were sequenced in Sangon Biotech (Shanghai, China).

The methods of sequence analysis and phylogenetic tree construction were described in the previous study (21). In short, the phylogenetic tree was conducted based on multiple sequence alignments using the Neighbor-Joining method in the MEGA 5.0 program.

\section{Tissue Distribution}

Three turbots were used and 12 tissues (skin, eye, liver, gill, brain, stomach, head kidney, intestine, adipose, spleen, heart and muscle) were isolated to analyze the foxo3 expression level in different tissues. Samples were kept stored at $-80^{\circ} \mathrm{C}$ until analysis.

\section{Immunofluorescence}

The immunofluorescence was used to analyze the distribution of FoxO3 in hepatocytes. In brief, a piece of liver tissue was taken out from a turbot, then washed with PBS and fixed in $4 \%$ paraformaldehyde at room temperature for $30 \mathrm{~min}$. Then, it was blocked for $1 \mathrm{~h}$ in $3 \%$ BSA, and followed by incubation with specific primary monoclonal rabbit anti-human antibody FoxO3 (1:200, AF609, Beyotime, China) (diluted with PBS appropriately) overnight at $4^{\circ} \mathrm{C}$. The suitability of the antibody FoxO3 for turbot has been verified prior to use (Supplementary Figures 1, 2). After that, the tissue was washed for three times with PBS for $5 \mathrm{~min}$ each time, followed by incubation with secondary antibody (Cy3 conjugated Goat Anti-Rabbit IgG) (1:200, GB21303, Servicebio, China) for $60 \mathrm{~min}$ at room temperature in the dark. Microscopy detection and images collection were by fluorescent microscopy (Eclipse C1, Nikon, Japan). The nucleus is blue by labeling with DAPI. Positive cells are red according to the fluorescent labels used.

\section{The Synthesis of siRNA and Its Knocking Down Efficiency}

Four siRNA duplexes (siRNA-485, siRNA-783, siRNA-1071, siRNA1349 , respectively) targeting different encoding regions of foxo 3 and a silence negative control siRNA (sifoxo3-NC) were designed and synthesized by Sangon (Sangon Biotech, China) (Table 2).

TABLE 2 | SiRNA sequences.

\begin{tabular}{|c|c|c|}
\hline Primer & Sense (5'-3') & Antisense (5'-3') \\
\hline sifoxo3-485 & GAGGTCCATCCCCTACTTCAA & UUGAAGUAGGGGAUGGACCUCTT \\
\hline sifoxo3-783 & GACAGTCCTTCAGGTCTCTCT & AGAGAGACCUGAAGGACUGUCTT \\
\hline sifoxo3-1071 & GGTACGATGAACCTGAATGAT & AUCAUUCAGGUUCAUCGUACCTT \\
\hline sifoxo3-1349 & GCAGACCATCCAGGAGAACAA & UUGUUCUCCUGGAUGGUCUGCTT \\
\hline sifoxo3-NC & UUCUCCGAACGUGUCACGUTT & ACGUGACACGUUCGGAGAATT \\
\hline
\end{tabular}


Primary hepatocytes were isolated from turbot liver and cultured as described previously (21). Cells were seeded in 6well plates (Corning, USA) at a density of $1.0 \times 10^{6}$ cells/well and incubated at $24^{\circ} \mathrm{C}$. When the cells were at $80-90 \%$ confluency, they were transfected with siRNAs. For transfection, $5 \mu \mathrm{g}$ of
siRNA and $3.75 \mu \mathrm{l}$ of Lipofectamine 3000 reagent (L3000015, Invitrogen, USA) were used in each well according to the manufacturer's protocol. Scrambled siRNA (sifoxo3-NC) served as a negative control for the experiment. No siRNA but an equal amount of PBS (Sangon Biotech, China) was added as a

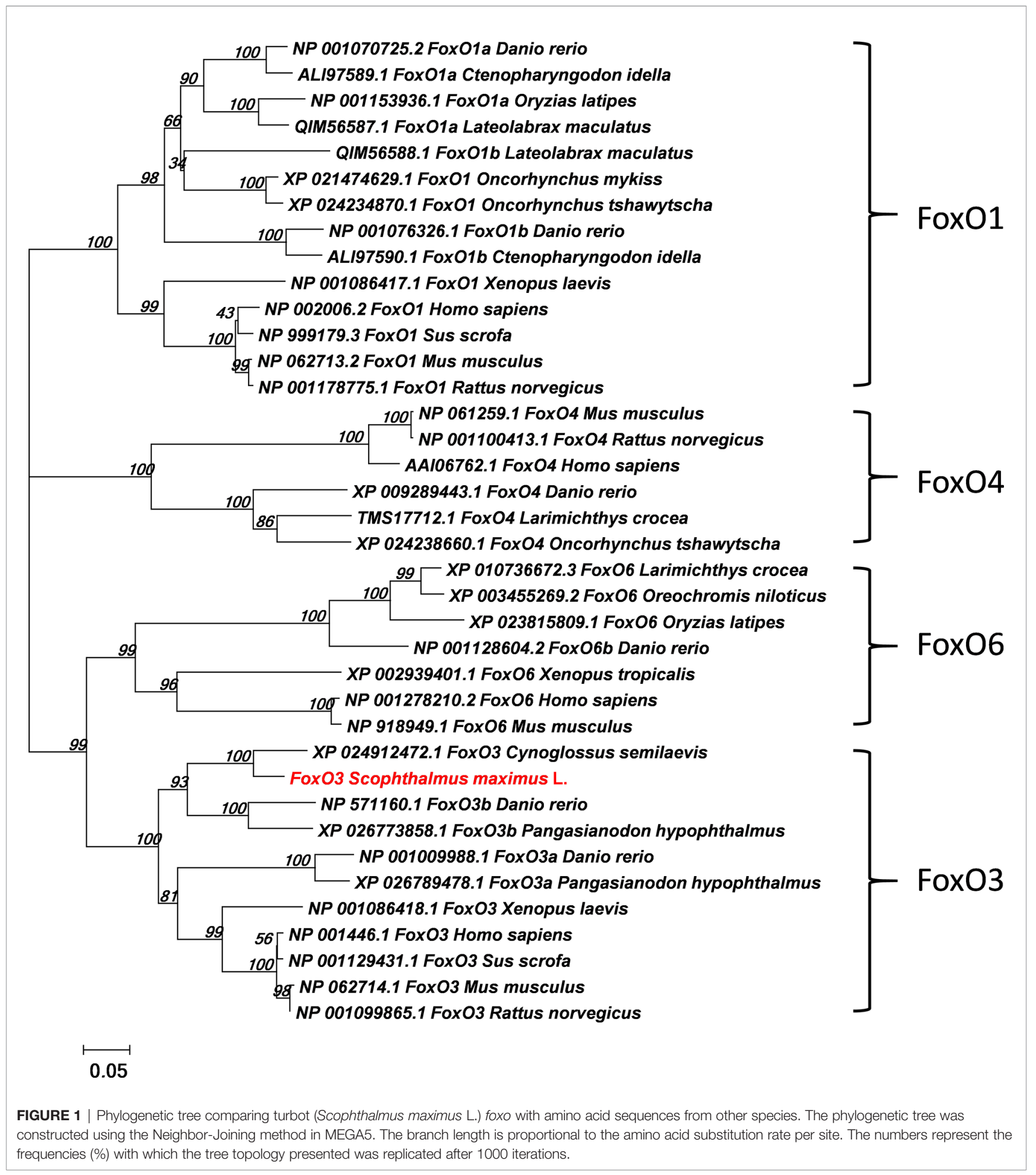


A

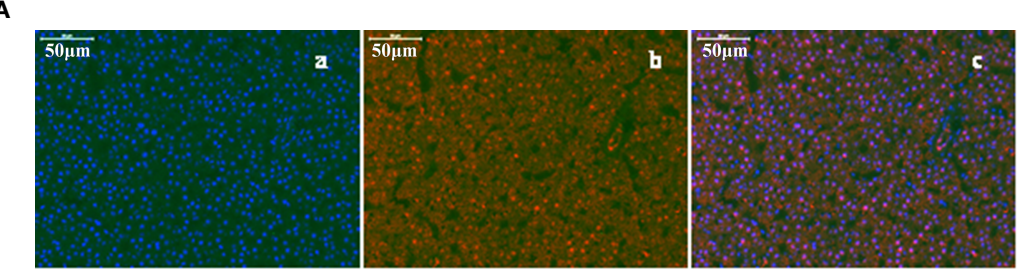

B

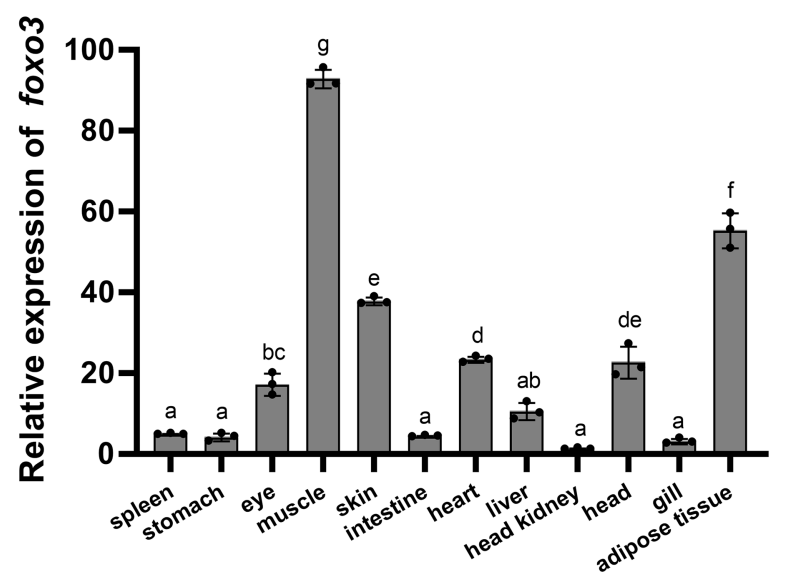

FIGURE 2 | (A) Immunofluorescent localization of FoxO3 in turbot liver. (a) DAPI staining; (b) FoxO3 staining; (c) Merge. (B) Expressions of foxo3 in different tissues of turbot. The results were expressed as the mean \pm standard deviation $(n=3)$. Bars bearing the different letters are significantly different between tissues by oneway analysis of variance (ANOVA) $(P<0.05)$.

control group. Cells were harvested after $24 \mathrm{~h}$ to determine transfection efficiency by qPCR. The transfection experiments were performed in triplicate. Experiments would be subsequently performed to assess the silence effects only if the transfection effects were $>50 \%$.

\section{Plasmid Construction}

A PCR product encoding the entire open reading frame (ORF) of foxo3 was cloned into the expression vector pcDNA3.1 (Invitrogen, USA). A single band of 1998 bp corresponding to the expected amplification product was obtained from 2.2. The template of foxo3 was PCR amplified using primers with homology arms to BamHI region in pcDNA3.1 consisting of the forward primer foxo3-bamh1-F: 5' - cttggtaccgagctcggatccAT GGCCGAGGCGCAGCTG-3' and the reverse primer foxo3bamh1-R: 5'-atggtggcgaccggtggatccGCCAGGTACCCAGCTCT GAGA-3'. The resulting amplicons were purified by gel electrophoresis and extracted using the SanPrep Column DNA Gel Extraction Kit (B110092, Sangon Biotech, China). The pcDNA3.1 plasmid was digested with enzyme BamHI
A

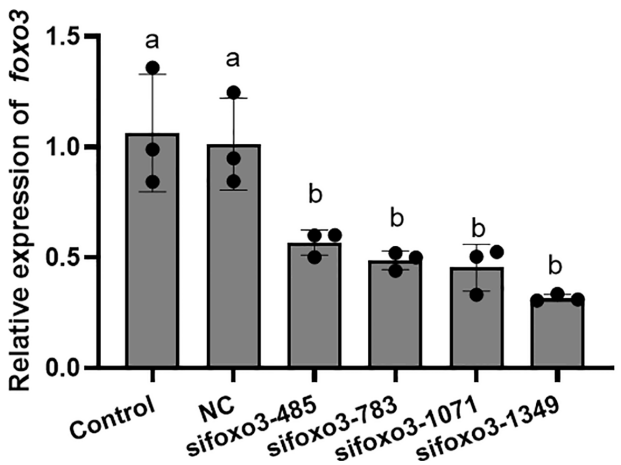

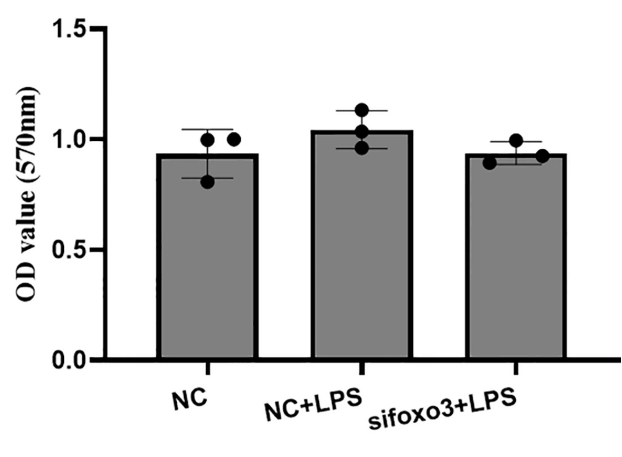

FIGURE 3 | (A) Relative level of foxo3 mRNA in primary cultured hepatocytes treated with sifoxo3. Results are represented as mean \pm standard deviation ( $\mathrm{n}=3$ ). Values with different letters mean significant differences $(P<0.05)$. (B) The OD values of MTT. Results are represented as mean \pm standard deviation ( $n=3)$. Values with different letters mean significant differences $(P<0.05)$. 
(D1010A, Takara, Japan). The purified resulting amplicons was then ligated into the pcDNA3.1 plasmid using ClonExpress Ultra One Step Cloning Kit (C112-02, Vazyme, China). The plasmid constructed was called pcDNA3.1-foxo3, and construct was confirmed by DNA sequencing. The pcDNA3.1-foxo3 plasmid was then transformed into Trans1-T1 Phage Resistant Chemically Competent Cell. After shake culture overnight, adequate plasmid for transfection was collected using a EasyPure HiPure Plasmid MaxiPrep Kit (EM121-01, TransGen, China).

\section{MTT Assay for Cell Viability}

For the determination of cell viability, primary hepatocytes were seeded in 96 well plates and treated with different kinds of treatments below. A MTT assay was performed to test cell viability according to standard protocols (M1020, Solarbio, China).

\section{LPS Stimulating and Knocking Down of Foxo3 In Vitro and In Vivo}

To study the effects of foxo3 in the biological behavior of LPSactivated hepatocellular inflammation, turbot primary hepatocytes were divided into three groups in 6 well plates: (1) NC: cells treated with one scrambled siRNA ( $5 \mu \mathrm{g} /$ well); (2) NC + LPS: cells treated with the scrambled siRNA (5 $\mu \mathrm{g} /$ well) and LPS (100 ng/ml); (3) sifoxo3 + LPS: cells treated with the sifoxo3 (5 $\mathrm{g} /$ well) and LPS $(100 \mathrm{ng} / \mathrm{ml})$. Cells were harvested after $24 \mathrm{~h}$ incubation and stored at $-80{ }^{\circ} \mathrm{C}$ for $\mathrm{qPCR}$ and Western Blot.

Injection test was used to examine the efficiency of the siRNA in vivo. The injection dose was determined by fish body weight. Two groups of turbot (12 fish/group) were intraperitoneally injected with $\mathrm{NC}(1 \mu \mathrm{g} / \mathrm{g})$ and sifoxo3 $(1 \mu \mathrm{g} / \mathrm{g})$, respectively. At $48 \mathrm{~h}$ post-injection, the expression of foxo3 in the liver was determined by RT-qPCR to guarantee foxo3 was knocked down successfully.

Thirty-six turbots were divided into three groups (12 fish/ group): (1) NC: turbots intraperitoneally injected with NC (1 $\mu \mathrm{g} /$ g) and PBS $(100 \mu \mathrm{l})$; (2) NC + LPS: turbots intraperitoneally injected with NC $(1 \mu \mathrm{g} / \mathrm{g})$ and LPS $(2.5 \mu \mathrm{g} / \mathrm{g})$; (3) sifoxo3 + LPS: turbots intraperitoneally injected with the sifoxo3 $(1 \mu \mathrm{g} / \mathrm{g})$ and LPS $(2.5 \mu \mathrm{g} / \mathrm{g})$. All the injection doses above were determined by fish body weight. The NC and sifoxo 3 were injected $24 \mathrm{~h}$ before injecting PBS and LPS. When turbots were intraperitoneally injected with PBS/LPS $24 \mathrm{~h}$ later, the serum and liver of turbots were collected and immediately frozen in liquid nitrogen. Three turbots were mixed together to make a sample.
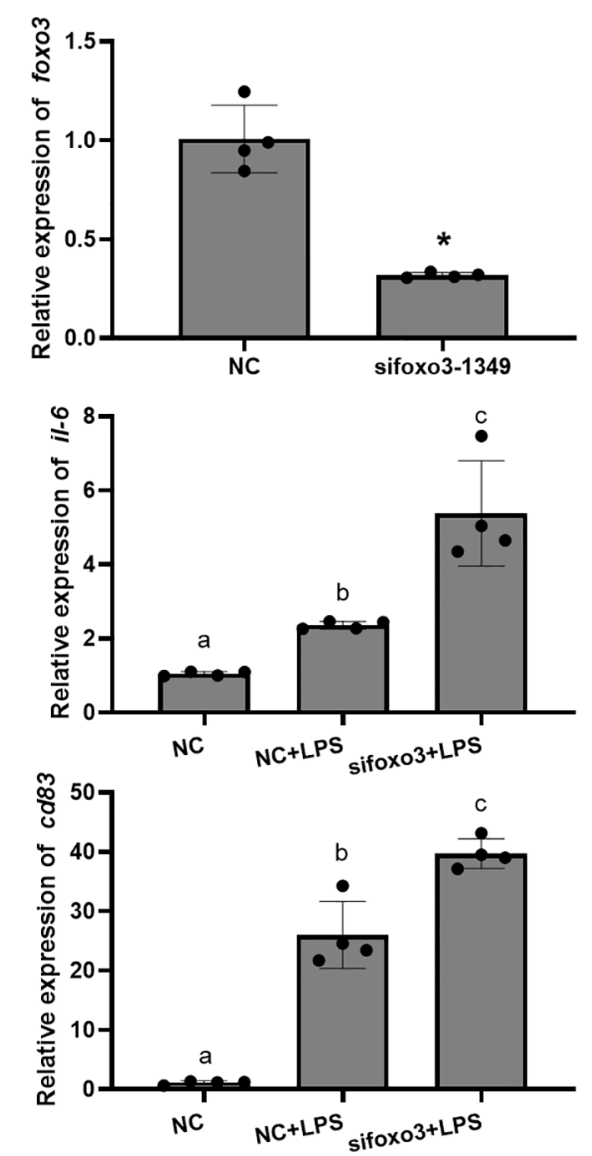
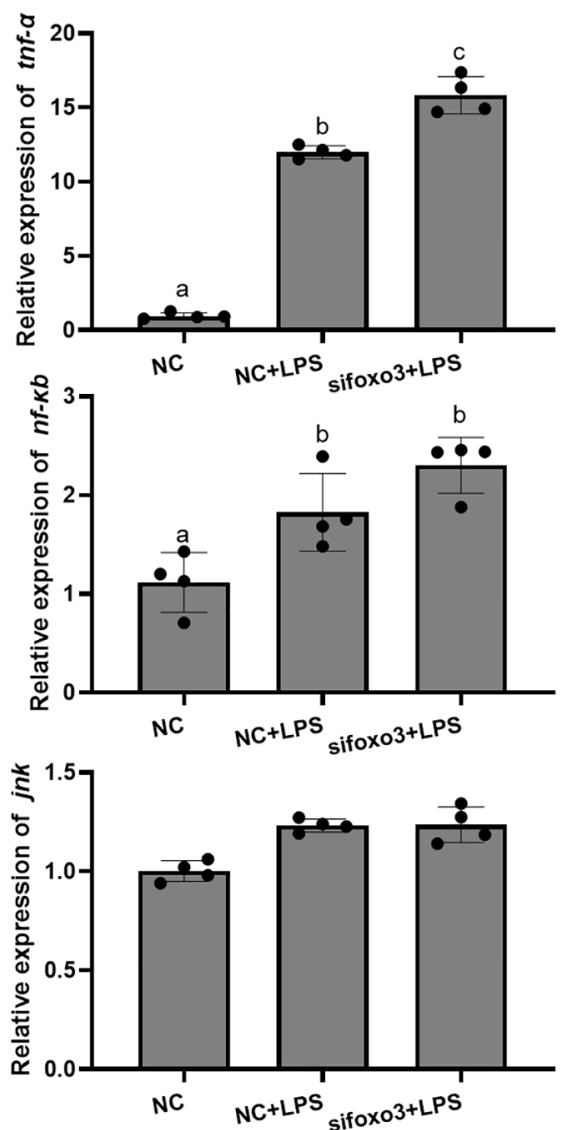
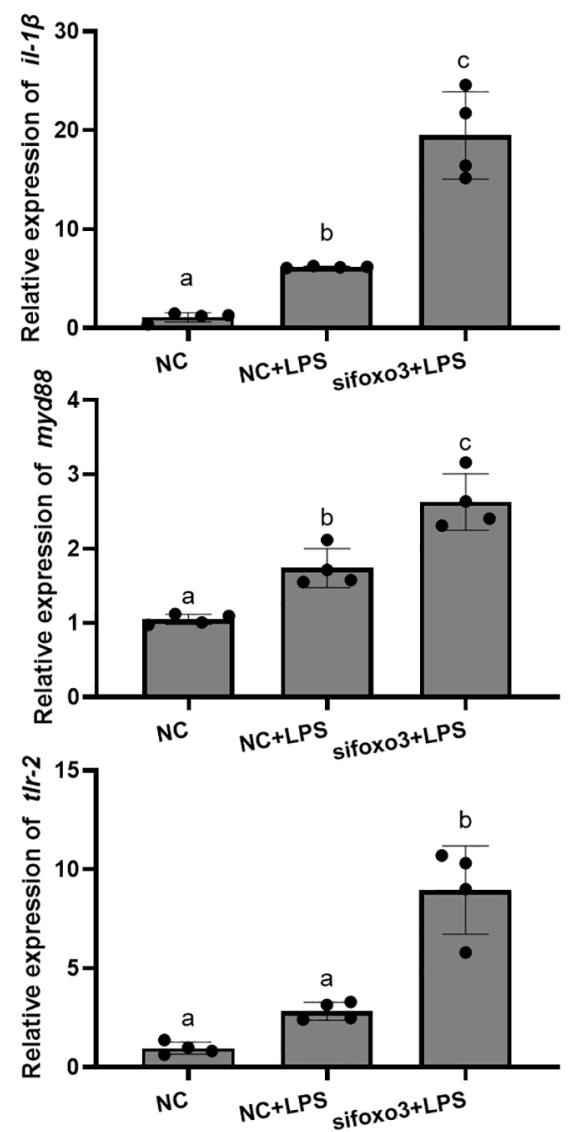

FIGURE 4 | Relative mRNA levels of foxo3, thf- $\alpha$, il-1 $\beta, i l-6, n f-\kappa b$, myd88, $c d 83$, jnk and tlr-2 in primary hepatocytes of turbot. The results are expressed as the mean \pm standard deviation $(n=4)$. '*' means significantly different between two group $(P<0.05)$. The different letters are significantly different $(P<0.05)$. 


\section{LPS Stimulating and Overexpression of Foxo3 In Vitro and In Vivo}

Turbot primary hepatocytes were seeded in 6 -well plates at a density of $1.0 \times 10^{6}$ cells/well and incubated at $24^{\circ} \mathrm{C}$. When the hepatocytes were at 80-90\% confluency, the cells were transfected with pcDNA3.1 or pcDNA3.1-foxo3 using Lipofectamine 3000 (L3000015, Invitrogen, USA) according to manufacturer's instruction. For each well, $5 \mu \mathrm{g}$ plasmid was used. Meanwhile, LPS was added into the wells after the plasmid transfection. There were three treatments respectively: (1) pcDNA3.1: cells treated with pcDNA3.1 (5 $\mu \mathrm{g} /$ well); (2) pcDNA3.1 + LPS: cells treated with pcDNA3.1 (5 $\mu \mathrm{g} /$ well $)$ and LPS (100 ng/ml); (3) pcDNA3.1-foxo3 + LPS: cells treated with the pcDNA3.1-foxo3 $(5 \mu \mathrm{g} /$ well $)$ and LPS (100 ng/ml). Cells were harvested after $24 \mathrm{~h}$ incubation and stored at $-80{ }^{\circ} \mathrm{C}$ for $\mathrm{RT}$ qPCR and Western Blot.

Prior to the overexpression of foxo3 in vivo, a pre-test was conducted to determine whether the plasmid could enter into liver of turbot intraperitoneally injected with pcDNA3.1-EGFP $(1 \mu \mathrm{g} / \mathrm{g})$ (Supplementary Figure 3). Injection dose was determined by fish body weight. After that, to examine the efficiency of the pcDNA3.1-foxo3 in vivo, two groups of turbot (12 fish/group) were intraperitoneally injected with pcDNA3.1 (1 $\mu \mathrm{g} / \mathrm{g})$ and pcDNA3.1-foxo3 $(1 \mu \mathrm{g} / \mathrm{g})$, respectively. At 48 h postinjection, the expression of foxo 3 in the liver was determined by RT-qPCR to guarantee foxo3 was upregulated successfully.

Thirty-six turbots of experiment were divided into three groups (12 fish/group): (1) pcDNA3.1, turbots intraperitoneally injected with pcDNA3.1 (1 $\mu \mathrm{g} / \mathrm{g})$ and PBS $(100 \mu \mathrm{l})$; (2) pcDNA3.1 + LPS: turbots intraperitoneally injected with pcDNA3.1 $(1 \mu \mathrm{g} / \mathrm{g})$ and LPS $(2.5 \mu \mathrm{g} / \mathrm{g})$; (3) pcDNA3.1-foxo3 + LPS: turbots intraperitoneally injected with the pcDNA3.1-foxo3 $(1 \mu \mathrm{g} / \mathrm{g})$ and LPS $(2.5 \mu \mathrm{g} / \mathrm{g})$. All the injection doses above were determined by fish body weight. The pcDNA3.1 and pcDNA3.1foxo3 were intraperitoneally injected $24 \mathrm{~h}$ before injecting PBS and LPS. When turbots were intraperitoneally injected with PBS/ LPS $24 \mathrm{~h}$ later, the serum and liver of turbots were collected and immediately frozen in liquid nitrogen. Three turbots were mixed together to make a sample.

\section{Serum Aminotransferase Activities}

Plasma samples were taken from the turbots at $24 \mathrm{~h}$ after PBS/ LPS injection described at 2.8 and 2.9. Serum levels of GPT (C009-2-1; Jiancheng, China), GOT (C010-2-1; Jiancheng, China) as markers of hepatic damage were measured performed according to the manufacturer's instructions.

\section{Quantitative Real-Time PCR (qPCR)}

The expression of target genes including foxo3, $\operatorname{tnf} \alpha, i l-1 \beta$, il-6, $n f-\kappa b, m y d 88, c d 83$, jnk and tlr 2 were detected according to the method described previously (21). Briefly, total RNA from samples was extracted with Trizol Reagent (Invitrogen, USA). The quality and quantity of RNA were assessed using agarose gel electrophoresis at $1.2 \%$ and spectrophotometric analysis (A 260:280 nm ratio). After that, complementary DNA (cDNA) was synthesized from $1 \mathrm{mg}$ of total RNA using the PrimeScript ${ }^{\text {TM }}$
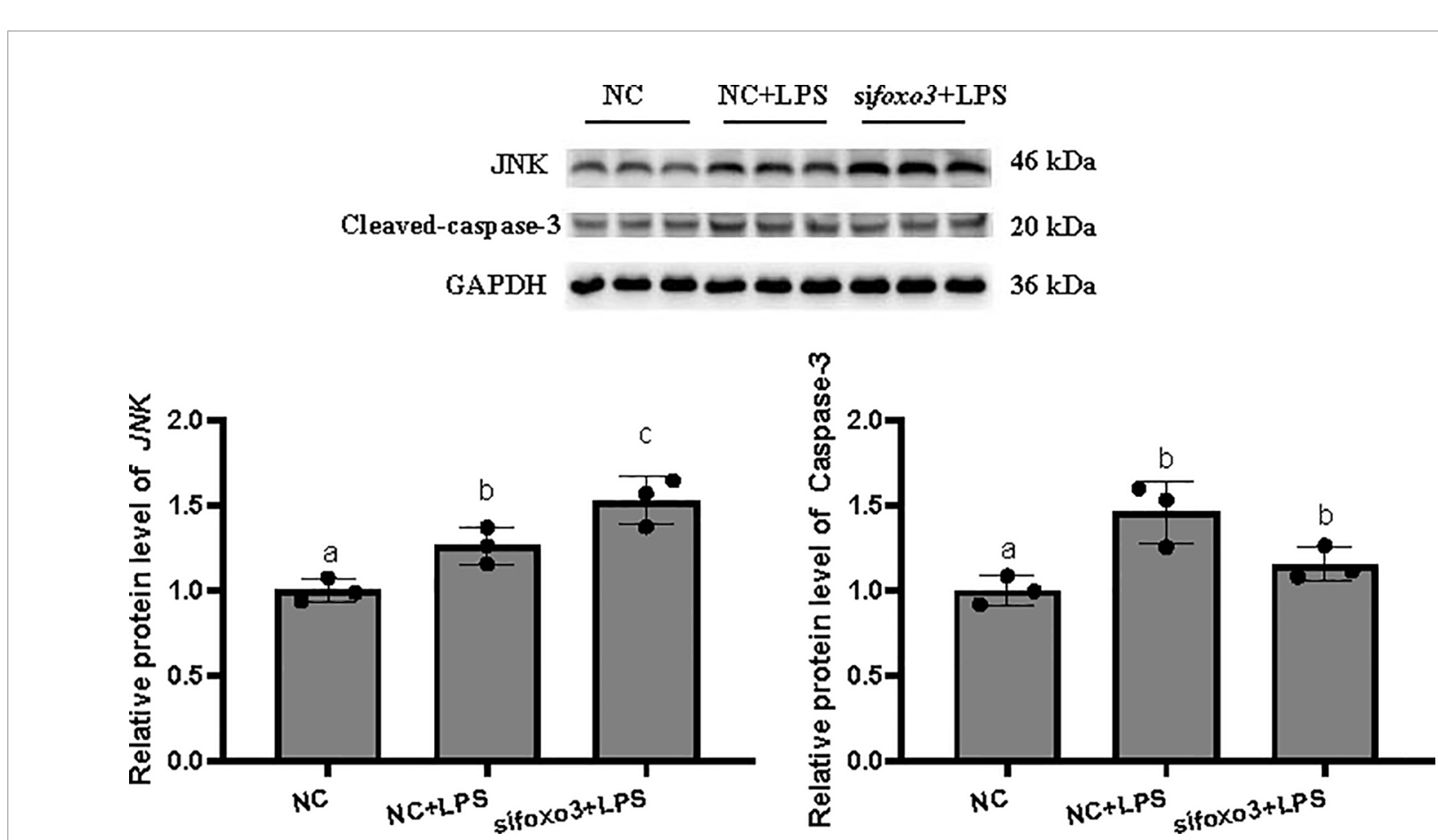

FIGURE 5 | Protein levels in turbot hepatocytes. The relative protein abundances of JNK and cleaved-caspase-3 in hepatocytes were measured by western blot and expressed as relative expression values to those in NC group. Values are represented as the mean \pm standard deviation ( $\mathrm{n}=3$ ). Values with different letters mean significantly difference $(P<0.05)$. 
RT reagent Kit with gDNA Eraser (RR047A, Takara, Japan). First strand cDNA was diluted 3 times using sterilized double-distilled water. qPCR was carried out in a quantitative thermal cycle (Quant Studio 5, Applied Biosystems, USA). The final reaction mixture contained $15 \mu \mathrm{L}$ containing $7.5 \mu \mathrm{L} 2 \times$ SYBR Green Realtime PCR Master Mix (Q711-02, Vazyme Biotech, China), $0.3 \mu \mathrm{L}$ each of primers $(10 \mu \mathrm{mol} / \mathrm{L})$ and $1 \mu \mathrm{L}$ of cDNA. $\beta$-actin was used as an endogenous reference to normalize the template amount. Real-time PCR temperature profile was $95^{\circ} \mathrm{C}$ for $30 \mathrm{~s}$ followed by $40 \mathrm{cycles}$ of $10 \mathrm{~s}$ at $95^{\circ} \mathrm{C}, 30 \mathrm{~s}$ at $58^{\circ} \mathrm{C}$. To confirm the specificity and purity of all PCR products, melt curve analysis was carried out after amplification: $95^{\circ} \mathrm{C}$ for $15 \mathrm{~s}, 60^{\circ} \mathrm{C}$ for $1 \mathrm{~min}$ and $95^{\circ} \mathrm{C}$ for $1 \mathrm{~s}$. The gene expressions were determined using the $2^{-\Delta \Delta \mathrm{Ct}}$. All the primers used in this study were listed in Table 1.

\section{Western Blot Analysis}

Protein homogenates preparation from turbot liver and hepatocytes, and western blotting were processed as refer to previous studies $(27,28)$. Equal amounts $(30 \mu \mathrm{g}$ per lane $)$ of protein were separated by sodium dodecyl sulfatepolyacrylamide gels (SDS-PAGE). Gels were cut according to molecular weight and transferred to $0.45 \mu \mathrm{m}$ PVDF membrane
(Millipore, Billerica, MA, USA). Different target proteins were tested in independent membrane, respectively. Incubation with the primary antibody was performed overnight at $4^{\circ} \mathrm{C}$. The primary antibodies used were polyclonal antibody rabbit antihuman JNK (1:500, WL05246, WanleiBio, China), polyclonal antibody rabbit anti-human cleaved-caspase-3 (1:500, WL02117, WanleiBio, China) and the polyclonal rabbit anti-human internal antibody control glyceraldehyde-3-phosphate dehydrogenase (GAPDH, 1:1000, AB-P-R001, Goodhere Biotechnology, China). After the incubation, the membrane was washed with TBST and incubated with secondary antibody (HRP-labeled goat anti-Rabbit lgG) (A0208, Beyotime, China) at 1:5000 dilution for $1 \mathrm{~h}$ at room temperature. The immune complexes were visualized using ECL reagents (P0018FM, Beyotime, China). The band densities were quantified using ImageJ software (National Institutes of Health, Bethesda, MD, USA). The suitability of the antibodies (JNK, cleaved-caspase-3 and GAPDH) for turbot has been verified prior to use (Supplementary Figure 4).

\section{Statistical Analysis}

All data were presented as means \pm S.D. (standard deviation) and showed all the individual data points. All statistical analyses were performed using SPSS 20 (IBM, IL, USA). The silencing
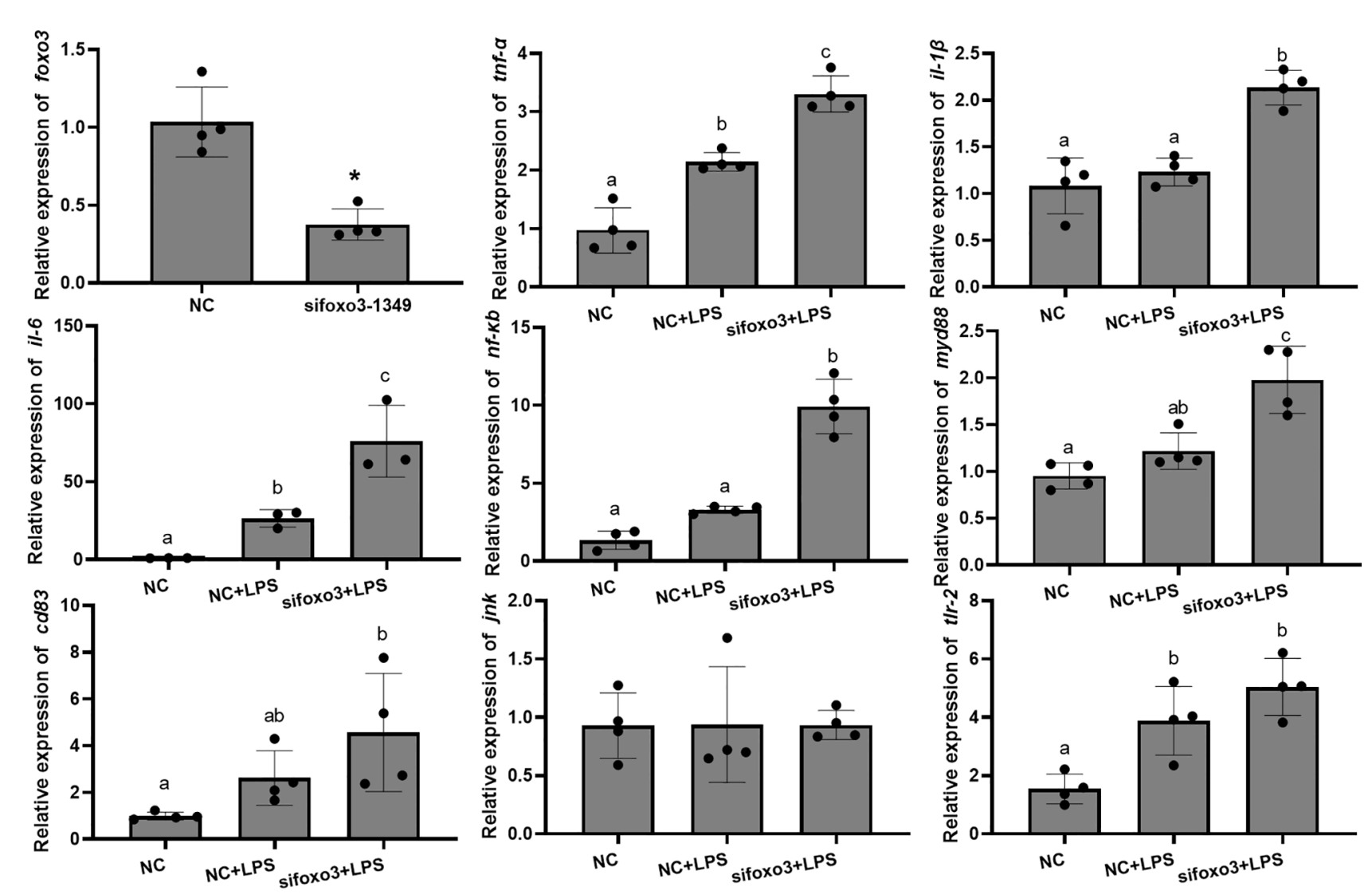

FIGURE 6 | Relative mRNA expression of foxo3, thf- $\alpha$, il-1 $\beta$, il-6, $n f-\kappa b$, myd88, cd83, jnk, tlr-2 in turbot liver. The results are expressed as the mean \pm standard deviation $(n=4)$. '*' means significantly different between two group $(P<0.05)$. The different letters are significantly different $(P<0.05)$. 
A

GPT

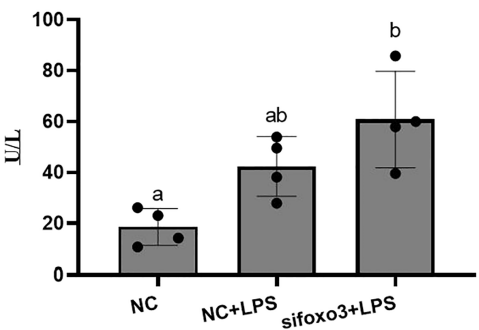

GOT

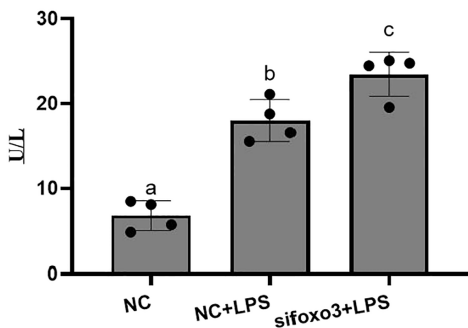

B

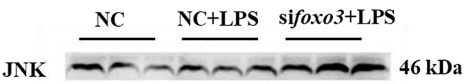

Cleaved-caspase-3 $3-20 \mathbf{m e g}$

GAPDH
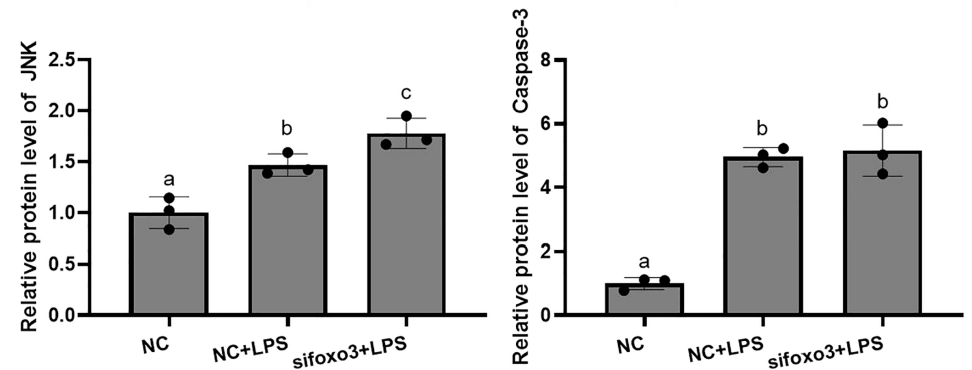

FIGURE 7 | (A) The levels of glutamate pyruvate transaminase (GPT) and glutamate oxaloacetate transaminase (GOT) in serum. Values are represented as the mean \pm standard deviation $(n=4)$. Values with different letters mean significantly difference $(P<0.05)$. (B) Protein levels in turbot liver. The relative protein abundances of JNK and cleaved-caspase-3 in turbot liver were measured by western blot and expressed as relative expression values to those in NC group. Values are represented as the mean \pm standard deviation $(n=3)$. Values with different letters mean significantly difference $(P<0.05)$.

efficiency of the siRNAs (sifoxo3-NC and sifoxo3-1349) and the overexpression efficiency of foxo3 (pcDNA3.1 and pcDNA3.1foxo3) in vitro and in vivo were analyzed by t-test. Other statistical evaluations were analyzed by one-way analysis of

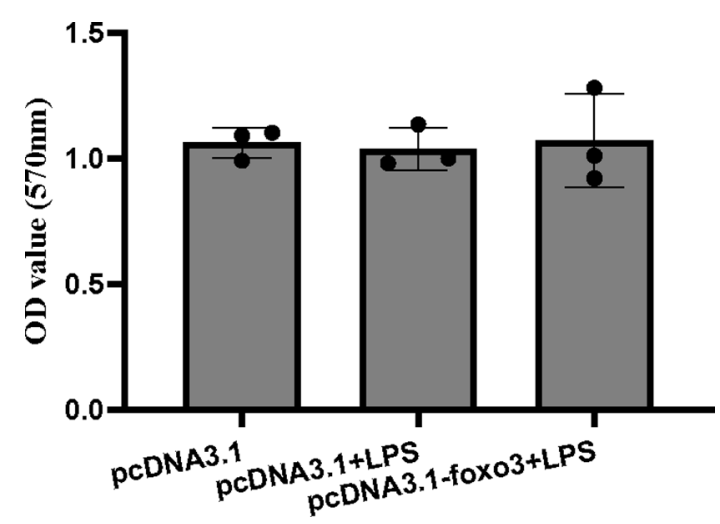

FIGURE 8 | The OD values of MTT. Results are represented as mean \pm standard deviation $(n=3)$. Values with different letters mean significant differences $(P<0.05)$. variance (ANOVA) followed by Tukey's multiple range tests. $P$-value $<0.05$ was considered to be statistically significant.

\section{RESULTS}

\section{Cloning, Characterization, Sequence Analysis and Tissue Distribution of Turbot Foxo3}

The full-length ORF of foxo3 was 1998 bp (GenBank Accession No: MW365446), encoding peptides of 665 amino acids with a predicted molecular weight of $70.06 \mathrm{kDa}$ and a theoretical isoelectric point of 4.80 . The phylogenetic tree was constructed with the full-length foxo amino acid of various species of foxol, foxo3, foxo 4 and foxo6 (Figure 1). The most similar species was tongue soles (Cynoglossus semilaevis). The immunofluorescent results showed that the $\mathrm{FoxO} 3$ expressed both in cytoplasm and nucleus (Figure 2A). The foxo 3 was broadly distributed in various tissues, and the abundance of mRNA varied among different tissues (Figure 2B). The expression level of foxo 3 gene was highest in the muscle, followed by adipose tissue, skin, heart, head, eye, liver, spleen, intestine, stomach, gill and head kidney, respectively. The gene expression of foxo3 in head kidney was the lowest relatively. 

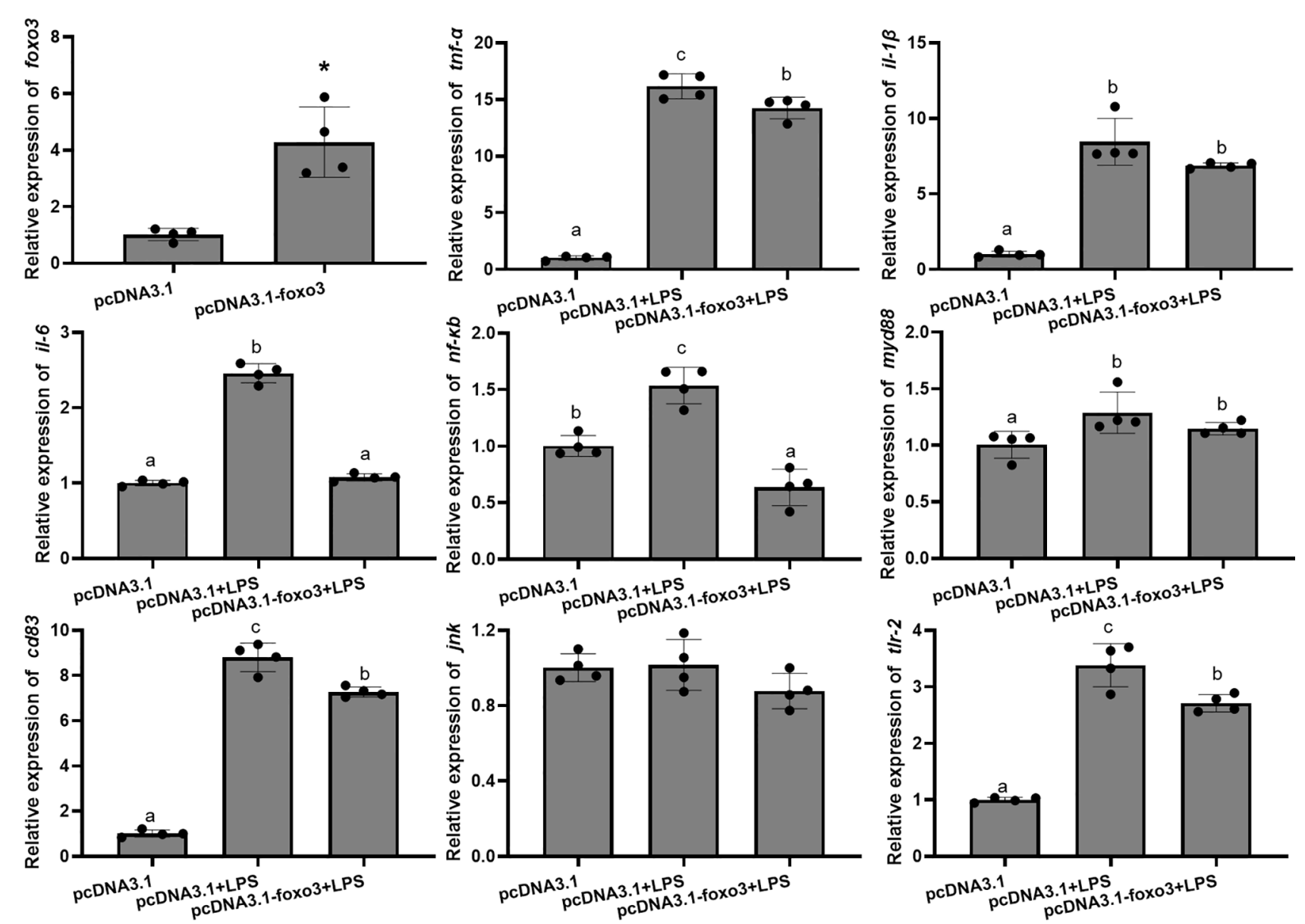

FIGURE 9 | Relative mRNA levels of foxo3, tnf- $\alpha$, il-1 $\beta, i l-6, n f-\kappa b$, myd88, cd83, jnk and tir-2 in the turbot primary hepatocytes. The results are expressed as the mean \pm standard deviation $(n=4)$. "*' means significantly different between two group $(P<0.05)$. The different letters are significantly different $(P<0.05)$.

\section{Inflammation-Related Genes and Proteins With Foxo3 Knocked Down In Vitro}

The result indicated that sifoxo3-1349 was the most efficient duplex for knocking down foxo3 expression in turbot liver cells (decreasing about 60\%) (Figure 3A), and this siRNA duplex was subsequently delivered in the following experiments. The cell viability was detected by MTT assay in the three groups. The result showed that there was no significant difference among the three groups $(P>0.05)$ (Figure 3B).

The mRNA levels of tnf- $\alpha, i l-1 \beta, i l-6, n f-\kappa b, m y d 88$ and $c d 83$ significantly increased after LPS incubation compared with the NC group $(P<0.05)$. The mRNA level of $j n k$ and $t l r-2$ increased after LPS incubation compared with the NC group, but there was no significant difference $(P>0.05)$. The mRNA levels of $\operatorname{tnf}-\alpha$, il$1 \beta$, il-6, myd88, $c d 83$ and $t l r-2$ significantly increased in the sifoxo3+LPS group compared with NC+LPS group $(P<0.05)$. The mRNA levels of $n f-\kappa b$ and $j n k$ increased in the sifoxo3+LPS group compared with the NC+LPS group, but there was no significant difference between the two groups $(P>0.05)$ (Figure 4).

The protein levels of JNK and cleaved-caspase-3 significantly increased after LPS incubation compared with the NC group $(P<$
$0.05)$. The protein level of JNK significantly increased in sifoxo3+ LPS group compared with the NC+LPS group $(P<0.05)$ and the protein level of cleaved-caspase-3 had no difference between the two groups $(P>0.05)$ (Figure 5).

\section{Inflammation-Related Genes, Enzyme Activity and Proteins With Foxo3 Knocked Down In Vivo}

To investigate the involvement of FoxO3 in LPS-induced liver inflammation in vivo, turbots were injected with specific small interfering in vivo. The result indicated that sifoxo3-1349 knocked down foxo3 expression in turbot liver cells (decreasing > 50\%) (Figure 6). The mRNA levels of $t n f-\alpha$, il-6, and tlr-2 significantly increased after LPS injection compared with NC group $(P<0.05)$. The mRNA levels of $i l-1 \beta, n f-\kappa b, m y d 88$ and $c d 83$ increased after LPS injection compared with NC group, but there was no significant difference $(P>0.05)$. The mRNA levels of $\operatorname{tnf}-\alpha, i l-1 \beta$, $i l-6, n f-\kappa b$ and myd88 significantly increased in sifoxo3+LPS group compared with NC+LPS group $(P<0.05)$. The mRNA levels of $c d 83$ and $t t r-2$ increased in the sifoxo3+LPS group compared with the NC+LPS group, but there was no significant difference between 

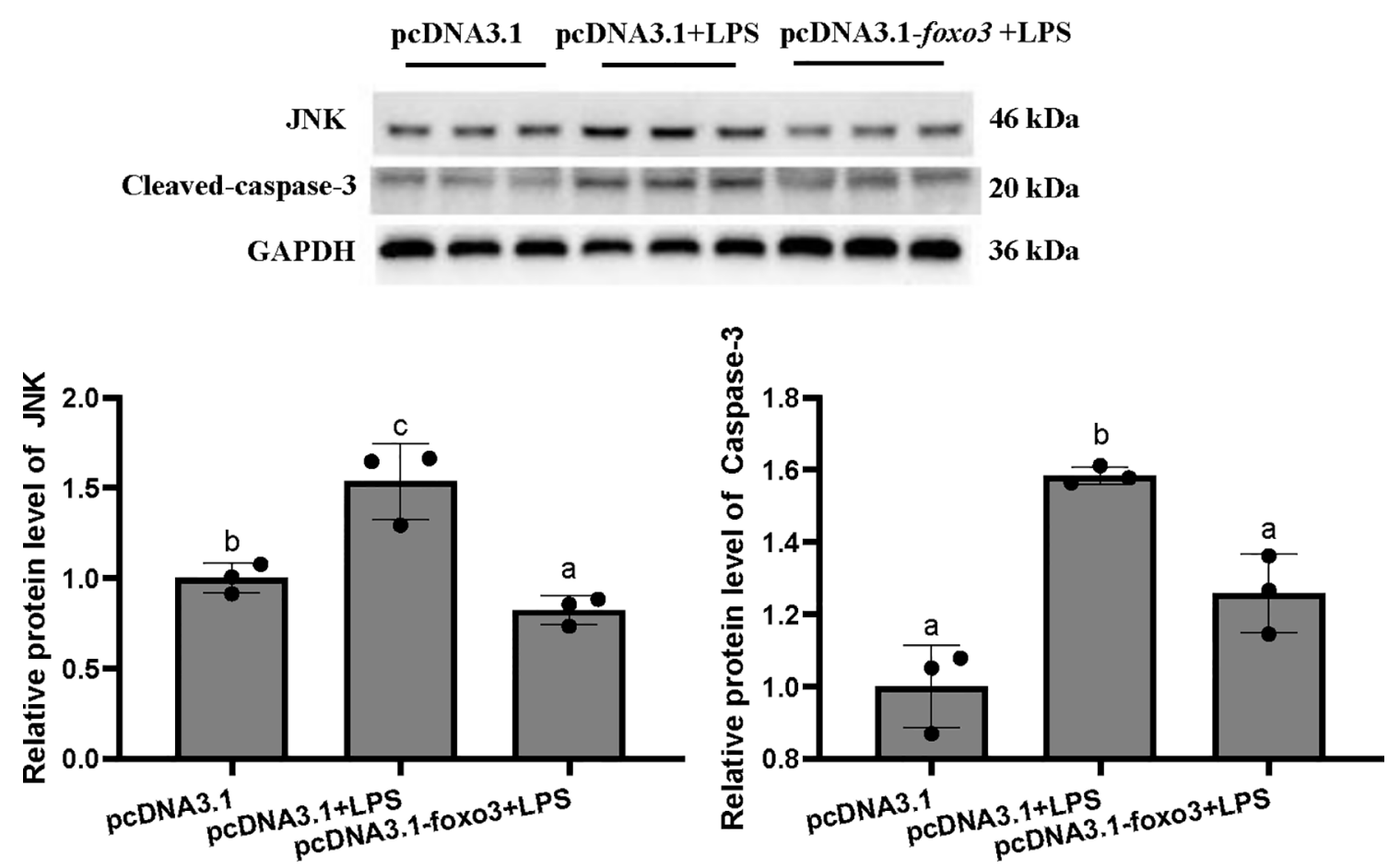

FIGURE 10 | Protein levels in turbot hepatocytes. The relative protein abundances of JNK and cleaved- caspase-3 in hepatocytes were measured by western blot and expressed as relative expression values to those in pcDNA3.1 group. Values are represented as means the mean \pm standard deviation $(n=3)$. Values with different letters mean significantly difference $(P<0.05)$.

the two groups $(P>0.05)$. The mRNA levels of $j n k$ remained unchanged among the three groups $(P>0.05)$ (Figure 6).

Results of GPT and GOT in serum showed that the level of GOT significantly increased after LPS injection compared with the NC group $(P<0.05)$. The level of GPT increased after LPS injection compared with the NC group. There was no significant difference between the two groups $(P>0.05)$. The level of GOT in serum significantly increased in sifoxo3+LPS group compared with NC+LPS group $(P<0.05)$ (Figure 7A). The level of GPT in serum increased in sifoxo3+LPS group compared with NC+LPS group, but there was no significant difference between the two groups $(P>0.05)$ (Figure 7A).

The protein levels of JNK and cleaved-caspase-3 significantly increased after LPS injection compared with NC group $(P<$ 0.05). The protein levels of JNK significantly increased in sifoxo3+ LPS group compared with NC+LPS group $(P<0.05)$ and the protein level of cleaved-caspase- 3 had no difference between the two groups $(P>0.05)$ (Figure 7B).

\section{Inflammation-Related Genes and Proteins With foxo3 Overexpressed In Vitro}

The MTT result showed that there was no significant difference among the pcDNA3.1 group, pcDNA3.1+LPS group and pcDNA3.1-foxo3+LPS group $(P>0.05)$ (Figure 8).

The mRNA levels of $t n f-\alpha, i l-1 \beta, i l-6, n f-\kappa b, m y d 88, c d 83$ and tlr-2 in each group significantly increased after LPS incubation compared with pcDNA3.1 group $(P<0.05)$. The mRNA levels of tnf- $\alpha, i l-6, n f-\kappa b, c d 83$ and $t l r-2$ significantly decreased in pcDNA3.1-foxo3+LPS group compared with pcDNA3.1+LPS group $(P<0.05)$. The mRNA levels of $i l-1 \beta$ and myd88 decreased in pcDNA3.1-foxo3+LPS group compared with pcDNA3.1+LPS group, but there was no significant difference between the two groups $(P>0.05)$. The mRNA levels of jnk remained unchanged among the three groups $(P>0.05)$ (Figure 9).

The protein levels of JNK and cleaved-caspase-3 significantly increased after LPS incubation compared with pcDNA3.1 group $(P<0.05)$. The protein levels of JNK and cleaved-caspase- 3 significantly decreased in pcDNA3.1-foxo3+LPS group compared with pcDNA3.1+LPS group $(P<0.05)$ (Figure 10).

\section{Inflammation-Related Genes, Enzyme Activity and Proteins With Foxo3 Overexpressed In Vivo}

To investigate the involvement of FoxO3 factor in LPS-induced liver inflammation in vivo, turbots injected with overexpression plasmid in vivo. The result indicated that pcDNA3.1-foxo3 upregulated foxo3 expression in turbot liver cells (increasing about $300 \%$ ) (Figure 11). The mRNA levels of $t n f-\alpha, i l-1 \beta$, il-6, $n f-\kappa b, m y d 88, c d 83, j n k$, and $t l r-2$ significantly increased after LPS injection compared with pcDNA3.1 group $(P<0.05)$. The mRNA levels of $i l-1 \beta$, il-6, myd88, cd83 and jnk significantly decreased in pcDNA3.1-foxo3+LPS group compared with pcDNA3.1+LPS group $(P<0.05)$. The mRNA levels of $\operatorname{tnf}-\alpha$, $n f-\kappa b$ and $t l r-2$ decreased in pcDNA3.1-foxo3+LPS group 


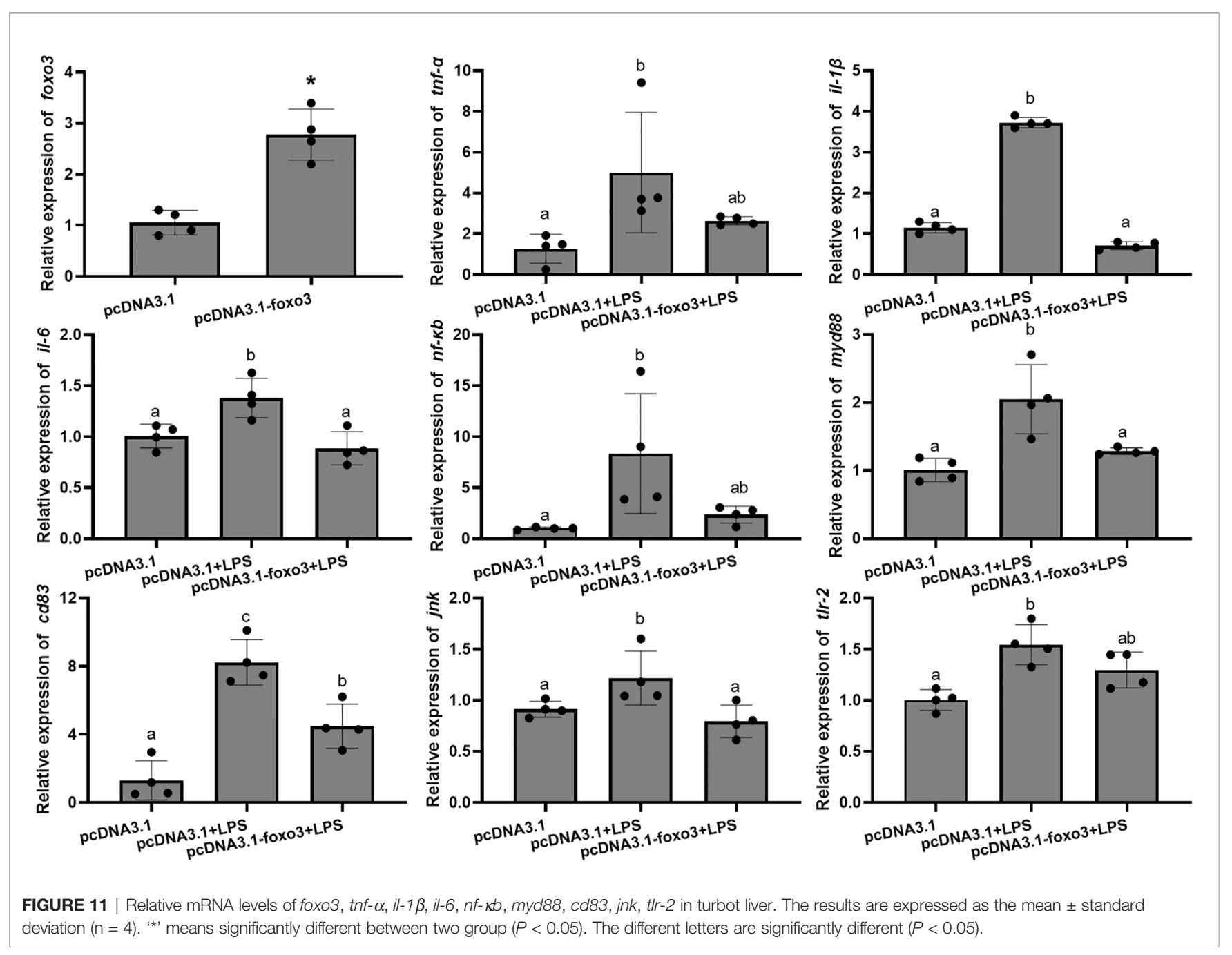

compared with pcDNA3.1+LPS group, but there was no significant difference between the two groups $(P>0.05)$ (Figure 11).

The results of GPT and GOT in serum showed that the level of GPT significantly increased after LPS injection compared with pcDNA3.1 group $(P<0.05)$ (Figure 12A). The level of GOT increased after LPS injection compared with pcDNA3.1 group, but there was no significant difference between the two groups $(P>0.05)$ (Figure 12A). The levels of GPT and GOT in serum significantly decreased in pcDNA3.1-foxo3+LPS group compared with pcDNA3.1+LPS group $(P<0.05)$ (Figure 12A).

The protein levels of JNK and cleaved-caspase- 3 significantly increased after LPS injection compared with pcDNA3.1 group $(P<0.05)$. The protein levels of JNK and cleaved-caspase- 3 significantly decreased in pcDNA3.1-foxo3+LPS group compared with pcDNA3.1+LPS group $(P<0.05)$ (Figure 12B).

\section{DISCUSSION}

The foxo3 belongs to the family of forkhead box $\mathrm{O}$ transcription factors and has emerged as an important role in immune regulation in mammals' liver $(17,29-31)$. In the present study, the foxo3 was cloned and characterized in turbot for the first time. The foxo3 mRNA was identified and the full-length ORF of foxo3 was $1998 \mathrm{bp}$, encoding peptides of 665 amino acids. In mammals, foxo3 is widely expressed in various tissues, including skeletal muscle, heart muscle, kidney, liver, pancreas, thymus and nervous system (32). In the present study, foxo3 was expressed in all the 12 analyzed tissues and the relative expression was highest in muscle, following by adipose tissue and skin. The relative expression of foxo3 was not the highest in turbot liver. However, the liver can produce defense regulators and mediators and has recently led to its perception as an important organ of innate immunity in mammals (33). In addition, the role of foxo3 in liver immunity and injury has been studied in many researches in other species $(17,30,34,35)$. Moreover, in the study of catfish, it was found that after $E$. ictaluri infection, foxo 3 was significantly upregulated in intestine, suggesting its involvement in responses bacterial infection in catfish intestine (20). In the present study, the relative expression of foxo 3 in liver was higher in intestine. Therefore, it was suspected that FoxO3 in liver may also play a crucial role in immune responses. This is the first time to report on the localization of foxo3 in fish. From the results of immunofluorescence, foxo3, as a 
A

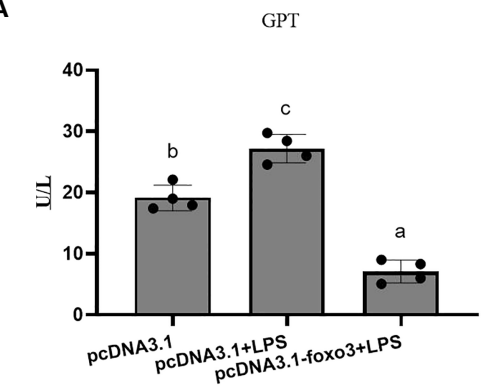

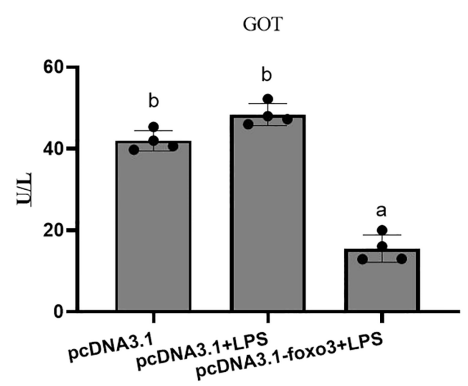

B
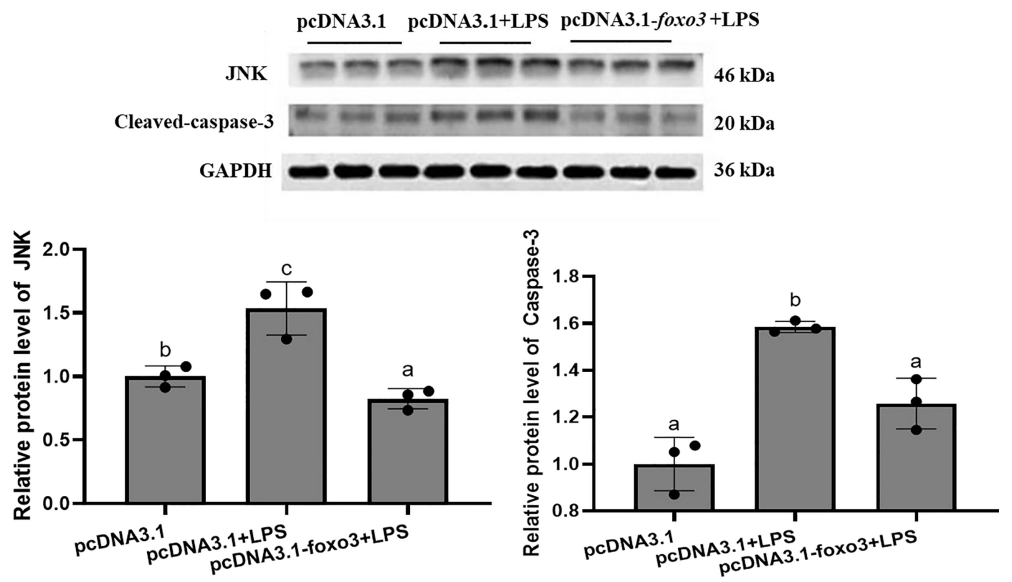

FIGURE 12 | (A) The levels of glutamate pyruvate transaminase (GPT) and glutamate oxaloacetate transaminase (GOT) in serum. Values are represented as the mean \pm standard deviation $(n=4)$. Values with different letters mean significantly difference $(P<0.05)$. (B) Protein levels in turbot liver. The relative protein abundances of JNK and cleaced-caspase-3 in turbot liver were measured by western blot and expressed as relative expression values to those in pcDNA3.1 group. Values are represented as the mean \pm standard deviation $(n=3)$. Values with different letters mean significantly difference $(P<0.05)$.

transcription factor, expressed in cytoplasm as well as in nucleus. Similar results were found in the study of human placenta and fetal membranes (36). In mammals, it was confirmed that the functions of foxo3 were related to changes in its cellular localization (37). However, further researches are needed in fish

LPS-induced liver injury has been used as experimental models to analyze the mechanism of endotoxin-induced acute liver injury $(38,39)$. LPS induces the release of numerous proinflammatory cytokines, including interleukin-1 (il-1), il-6, and tumor necrosis factor $(t n f)$ both in mammals and fish $(6,40-$ 42). Hence, LPS incubated in vitro and injected in vivo were used to construct inflammatory models. In the present study, the relative expressions of $i l-1 \beta$, il-6 and $t n f \alpha$ increased after LPS administration both in vivo and in vitro. Meanwhile, knockdown of foxo3 led to significant elevating of these mRNA levels, while the foxo 3 overexpression significantly decreased these levels. This is consistent with the previous study, in which foxo 3 overexpression in intestinal epithelial cells caused a significant decrease in the gene expressions of $i l-1$ and $i l-6$ (13). In mice, silence of foxo 3 increased the gene expressions of $i l-1 \beta$, il- 6 and $\operatorname{tnf} \alpha$, but overexpressing of foxo3 attenuated liver failure by decreasing the mRNA levels of those genes (16). Therefore, the conclusion can be drawn that foxo3 can suppress inflammation by regulating proinflammatory cytokines.
GPT and GOT in serum are markers of hepatic damage, and the GPT and GOT levels in serum were increased in liver failure patients (43). In addition, when fish were exposed to environmental toxins, the serum levels of GPT and GOT increased significantly (44). Moreover, when juvenile red sea bream (Pagrus major) fed with the diet with excessive amounts of nucleoside by-products, the serum level of GPT increased significantly, which consistent with liver damage (45). Therefore, increased GPT and GOT levels were considered to indicate hepatocyte injury or liver damage in fish. In the present study, when turbots were injected with LPS $(2.5 \mu \mathrm{g} / \mathrm{g})$, the levels of GPT and GOT were increased. The results showed that LPS could cause liver damage in turbot. When the turbots were injected with sifoxo 3 for $24 \mathrm{~h}$ before the turbots were injected with LPS, the levels of GPT and GOT in serum increased compared with the NC+LPS group. It was indicated that inhibition of foxo3 in vivo increased LPS-activated liver damage in turbot. However, when the turbots were injected with pcDNA3.1-foxo3 for $24 \mathrm{~h}$ before the turbots were injected with LPS, the levels of GPT and GOT in serum decreased compared with the pcDNA3.1+LPS group. It was indicated that overexpression of foxo 3 in vivo relieved LPS-activated liver injury in turbot. Therefore, it is suggested that foxo 3 can relieve liver damage induced by LPS. 
JNK has been implicated in involving in liver damage (46) and triggering the activation of foxo3 in response to immune stress $(47,48)$. In breast cancer cells, cardamonin induces apoptosis via activation of the JNK/FoxO3 pathway (49). According to the report of Yang et al. (50), FoxO3 via ROS/ JNK/FoxO3 pathway plays a critical role to function as negative regulator associating with deoxynivalenol-induced cytotoxicity, with the potential extending to other substances (50). Therefore, there is a close relationship between JNK and FoxO3 when they play the role in immune regulation. In the present study, knock down of foxo 3 could not change the gene expressions of $j n k$, but increased the protein levels of JNK both in vivo and vitro. The results indicated that JNK was affected by foxo 3 at the protein level rather than the gene expression level in turbot liver cells. Furthermore, it has been confirmed that FoxO3/JNK pathway can regulate apoptosis (51-53), so the protein levels of cleavedcaspase- 3 were detected in the present study. The present results showed that the protein levels of cleaved-caspase- 3 had not been changed after foxo3 knocking down. Interestingly, the protein levels of JNK and cleaved-caspase- 3 showed the same trend when overexpression of foxo 3 both in vitro and in vivo, which revealed that foxo3 was a negatively regulated transcription factor in the JNK and caspase- 3 mediated pathways. Those results indicated foxo 3 regulated immune function through both caspase- 3 dependent and independent in JNK/caspase-3 pathway.

It was reported that $t l r-2$ was a major mediator of the inflammatory response, which associated with inflammation and liver injury tightly (54-56). Ma et al. (57) reported that tlr2 could activate the $n f-\kappa b$ pathway to regulate inflammatory response in a myd88-dependent pathway (57). Interestingly, the phenomenon was confirmed in the present study, in which the mRNA levels of $t / r 2, m y d 88$ and $n f-\kappa b$ were up-regulated after LPS incubating. In addition, knockdown of foxo 1 and foxo 3 increased endogenous $t l r-2$ mRNA levels. It was suggested that foxo1/3 suppresses tlr-2 in keratinocytes (58). In the present study, the results showed that knockdown of foxo3 both in vitro and in vivo could up-regulate the mRNA levels of tlr-2, myd88 and $n f-\kappa b$. Moreover, overexpression of foxo 3 both in vitro and in vivo could reduce the mRNA levels of $t / r 2$, myd88 and $n f-\kappa b$. These results indicated that LPS could activated $t / r-2 / \mathrm{myd} 88 / n f$ $\kappa b$ pathway and foxo3 inhibit LPS-induced inflammation through this signal pathway.

To sum up, the full-length ORF of foxo3 sequence was cloned from turbot. FoxO3 overexpression can inhibit LPS-induced inflammation in vivo and vitro by decreasing proinflammatory cytokines, the levels of GOT and GPT as well activating JNK/

\section{REFERENCES}

1. Zhang K, Li L, Qi Y, Zhu X, Gan B, DePinho RA, et al. Hepatic Suppression of Foxo1 and Foxo3 Causes Hypoglycemia and Hyperlipidemia in Mice. Endocrinology (2012) 153(2):631-46. doi: 10.1210/en.2011-1527

2. Tikhanovich I, Cox J, Weinman SA. Forkhead Box Class O Transcription Factors in Liver Function and Disease. J Gastroenterol Hepatol (2013) 28 Suppl 1(s1):125-31. doi: 10.1111/jgh.12021

3. Tong W, Ju L, Qiu M, Xie Q, Chen Y, Shen W, et al. Liraglutide Ameliorates non-Alcoholic Fatty Liver Disease by Enhancing Mitochondrial Architecture caspase-3 and $t l r-2 / m y d 88 / n f-\kappa b$ pathways. FoxO3 knocking down can activate LPS-induced inflammation through above signaling pathways in vivo and vitro. The research of FoxO3 in liver immunity in turbot enriches the studies on immune regulation in fish, and provides theoretical basis and molecular targets for solving liver inflammation and liver injury in fish.

\section{DATA AVAILABILITY STATEMENT}

The datasets presented in this study can be found in online repositories. The names of the repository/repositories and accession number(s) can be found in the article/Supplementary Material.

\section{ETHICS STATEMENT}

The animal study was reviewed and approved by Institutional Animal Care and Use Committee of Ocean University of China. Written informed consent was obtained from the owners for the participation of their animals in this study.

\section{AUTHOR CONTRIBUTIONS}

MP: Data curation, analysis and writing original draft. JL, DH, YG, KL, and MY: Data curation. WG and QX: Conceptualization. WZ: Conceptualization, funding acquisition, methodology, supervision, writing review and editing. KM: Methodology. All authors contributed to the article and approved the submitted version.

\section{FUNDING}

This study was financially supported by the National Key $\mathrm{R}$ \& D Program of China (grant number: 2019YFD09002 00, 2018YFD0900400).

\section{SUPPLEMENTARY MATERIAL}

The Supplementary Material for this article can be found online at: https://www.frontiersin.org/articles/10.3389/fimmu.2021.679704/ full\#supplementary-material

and Promoting Autophagy Through the SIRT1/SIRT3-FOXO3a Pathway. Hepatol Res (2016) 46(9):933-43. doi: 10.1111/hepr.12634

4. Pan X, Zhang Y, Kim HG, Liangpunsakul S, Dong XC. FOXO Transcription Factors Protect Against the Diet-Induced Fatty Liver Disease. Sci Rep (2017) 7 (1):44597. doi: $10.1038 /$ srep44597

5. Dong XC. FOXO Transcription Factors in Non-Alcoholic Fatty Liver Disease. Liver Res (2017) 1(3):168-73. doi: 10.1016/j.livres.2017.11.004

6. Yue Y, Wang Y, Li D, Song Z, Jiao H, Lin H. A Central Role for the Mammalian Target of Rapamycin in LPS-Induced Anorexia in Mice. J Endocrinol (2015) 224(1):37-47. doi: 10.1530/JOE-14-0523 
7. You H, Pellegrini M, Tsuchihara K, Yamamoto K, Hacker G, Erlacher M, et al. FOXO3a-Dependent Regulation of Puma in Response to Cytokine/Growth Factor Withdrawal. J Exp Med (2006) 203(7):1657-63. doi: 10.1084/jem.20060353

8. Myatt SS, Lam EW. The Emerging Roles of Forkhead Box (Fox) Proteins in Cancer. Nat Rev Cancer (2007) 7(11):847-59. doi: 10.1038/nrc2223

9. Mei Y, Wang Z, Zhang L, Zhang Y, Li X, Liu H, et al. Regulation of Neuroblastoma Differentiation by Forkhead Transcription Factors FOXO1/ 3/4 Through the Receptor Tyrosine Kinase PDGFRA. Proc Natl Acad Sci USA (2012) 109(13):4898-903. doi: 10.1073/pnas.1119535109

10. Zhang Y, Xing Y, Zhang L, Mei Y, Yamamoto K, Mak TW, et al. Regulation of Cell Cycle Progression by Forkhead Transcription Factor FOXO3 Through Its Binding Partner DNA Replication Factor Cdt1. Proc Natl Acad Sci USA (2012) 109(15):5717-22. doi: 10.1073/pnas.1203210109

11. Bullock MD, Bruce A, Sreekumar R, Curtis N, Cheung T, Reading I, et al. FOXO3 Expression During Colorectal Cancer Progression: Biomarker Potential Reflects a Tumour Suppressor Role. Br J Cancer (2013) 109 (2):387-94. doi: 10.1038/bjc.2013.355

12. Lu YF, Yu JR, Yang Z, Zhu GX, Gao P, Wang H, et al. Promoter Hypomethylation Mediated Upregulation of MicroRNA-10b-3p Targets FOXO3 to Promote the Progression of Esophageal Squamous Cell Carcinoma (ESCC). J Exp Clin Cancer Res (2018) 37(1):301. doi: 10.1186/s13046-018-0966-1

13. Yin Y, Wang J, Zhao X, Wu X, Zou H, Qin Z, et al. Overexpressed FOXO3 Improves Inflammatory Status in Mice by Affecting NLRP3-Mediated Cell Coronation in Necrotizing Colitis Mice. BioMed Pharmacother (2020) 125:109867. doi: 10.1016/j.biopha.2020.109867

14. Karpenko MN, Vasilishina AA, Gromova EA, Muruzheva Z, Miliukhina IV, Bernadotte A. Interleukin-1beta, Interleukin-1 Receptor Antagonist, Interleukin-6, Interleukin-10, and Tumor Necrosis Factor-Alpha Levels in CSF and Serum in Relation to the Clinical Diversity of Parkinson's Disease. Cell Immunol (2018) 327:77-82. doi: 10.1016/j.cellimm.2018.02.011

15. Dhainaut JF, Marin N, Mignon A, Vinsonneau C. Hepatic Response to Sepsis: Interaction Between Coagulation and Inflammatory Processes. Crit Care Med (2001) 29(7 Suppl):S42-47. doi: 10.1097/00003246-200107001-00016

16. Liu YM, Lv J, Zeng QL, Shen S, Xing JY, Zhang YY, et al. AMPK Activation Ameliorates D-GalN/LPS-Induced Acute Liver Failure by Upregulating Foxo3A to Induce Autophagy. Exp Cell Res (2017) 358(2):335-42. doi: 10.1016/j.yexcr.2017.07.008

17. Li Z, Zhao J, Zhang S, Weinman SA. FOXO3-Dependent Apoptosis Limits Alcohol-Induced Liver Inflammation by Promoting Infiltrating Macrophage Differentiation. Cell Death Discov (2018) 4(1):16. doi: 10.1038/s41420-017-0020-7

18. Zeng L, Ai CX, Zhang JS, Li WC. Pre-Hypoxia Exposure Inhibited Copper Toxicity by Improving Energy Metabolism, Antioxidant Defence and Mitophagy in the Liver of the Large Yellow Croaker Larimichthys crocea. Sci Total Environ (2020) 708:134961. doi: 10.1016/j.scitotenv.2019.134961

19. Zhao Y, Li JY, Jiang Q, Zhou XQ, Feng L, Liu Y, et al. Leucine Improved Growth Performance, Muscle Growth, and Muscle Protein Deposition Through AKT/TOR and AKT/FOXO3a Signaling Pathways in Hybrid Catfish. Pelteobagrus vachelli $\times$ Leiocassis Longirostris Cells (2020) 9(2):327. doi: 10.3390/cells 9020327

20. Gao L, Yuan Z, Zhou T, Yang Y, Gao D, Dunham R, et al. FOXO Genes in Channel Catfish and Their Response After Bacterial Infection. Dev Comp Immunol (2019) 97:38-44. doi: 10.1016/j.dci.2019.03.010

21. Pan MZ, Zhang Y, Deng KY, Liu GX, Gu ZX, Liu JH, et al. Forkhead Box O1 in Turbot Scophthalmus Maximus: Molecular Characterization, Gene Structure, Tissue Distribution and the Role in Glucose Metabolism. Gene (2019) 708:49-56. doi: 10.1016/j.gene.2019.03.065

22. Montes M, Farto R, Perez MJ, Nieto TP, Larsen JL, Christensen H. Characterization of Vibrio Strains Isolated From Turbot (Scophthalmus Maximus) Culture by Phenotypic Analysis, Ribotyping and 16S rRNA Gene Sequence Comparison. J Appl Microbiol (2003) 95(4):693-703. doi: 10.1046/ j.1365-2672.2003.02028.x

23. Castro N, Toranzo AE, Barja JL, Nunez S, Magarinos B. Characterization of Edwardsiella Tarda Strains Isolated From Turbot, Psetta maxima (L.). J Fish Dis (2006) 29(9):541-7. doi: 10.1111/j.1365-2761.2006.00750.x

24. Liu F, Su B, Fu Q, Shang M, Gao C, Tan F, et al. Identification, Characterization and Expression Analysis of TLR5 in the Mucosal Tissues of Turbot (Scophthalmus Maximus L.) Following Bacterial Challenge. Fish Shellfish Immunol (2017) 68:272-9. doi: 10.1016/j.fsi.2017.07.021
25. Ribas L, Pardo BG, Fernandez C, Alvarez-Dios JA, Gomez-Tato A, Quiroga MI, et al. A Combined Strategy Involving Sanger and 454 Pyrosequencing Increases Genomic Resources to Aid in the Management of Reproduction, Disease Control and Genetic Selection in the Turbot (Scophthalmus Maximus). BMC Genomics (2013) 14(1):180. doi: 10.1186/1471-2164-14-180

26. Zhang J, Xu X, Zhu H, Wang Y, Hou Y, Liu Y. Dietary Fish Oil Supplementation Alters Liver Gene Expressions to Protect Against LPSInduced Liver Injury in Weanling Piglets. Innate Immun (2019) 25(1):6072. doi: $10.1177 / 1753425918821420$

27. Deng KY, Pan MZ, Liu JH, Yang MX, Gu ZX, Zhang Y, et al. Chronic Stress of High Dietary Carbohydrate Level Causes Inflammation and Influences Glucose Transport Through SOCS3 in Japanese Flounder. Paralichthys Olivaceus Sci Rep (2018) 8(1):7415. doi: 10.1038/s41598-018-25412-w

28. Dong YZ, Li L, Espe M, Lu KL, Rahimnejad S. Hydroxytyrosol Attenuates Hepatic Fat Accumulation via Activating Mitochondrial Biogenesis and Autophagy Through the AMPK Pathway. J Agric Food Chem (2020) 68 (35):9377-86. doi: 10.1021/acs.jafc.0c03310

29. Notas G, Alexaki VI, Kampa M, Pelekanou V, Charalampopoulos I, SabourAlaoui S, et al. APRIL Binding to BCMA Activates a JNK2-FOXO3-GADD45 Pathway and Induces a G2/M Cell Growth Arrest in Liver Cells. J Immunol (2012) 189(10):4748-58. doi: 10.4049/jimmunol.1102891

30. Tumurbaatar B, Tikhanovich I, Li Z, Ren J, Ralston R, Kuravi S, et al. Hepatitis $\mathrm{C}$ and Alcohol Exacerbate Liver Injury by Suppression of FOXO3. Am J Pathol (2013) 183(6):1803-14. doi: 10.1016/j.ajpath.2013.08.013

31. Liu JH, Cao L, Zhang CH, Li C, Zhang ZH, Wu Q. Dihydroquercetin Attenuates Lipopolysaccharide-Induced Acute Lung Injury Through Modulating FOXO3-Mediated NF-KappaB Signaling via miR-132-3p. Pulm Pharmacol Ther (2020) 64:101934. doi: 10.1016/j.pupt.2020.101934

32. Luo N, Gao HM, Wang YQ, Li HJ, Li Y. MiR-942-5p Alleviates Septic Acute Kidney Injury by Targeting FOXO3. Eur Rev Med Pharmacol Sci (2020) 24 (11):6237-44. doi: 10.26355/eurrev_202006_21521

33. Nemeth E, Baird AW, O'Farrelly C. Microanatomy of the Liver Immune System. Semin Immunopathol (2019) 31(3):333-43. doi: 10.1007/s00281-0090173-4

34. Tao GZ, Lehwald N, Jang KY, Baek J, Xu B, Omary MB, et al. Wnt/betaCatenin Signaling Protects Mouse Liver Against Oxidative Stress-Induced Apoptosis Through the Inhibition of Forkhead Transcription Factor Foxo3. J Biol Chem (2013) 288(24):17214-24. doi: 10.1074/jbc.M112.445965

35. Piccolo P, Ferriero R, Barbato A, Attanasio S, Monti M, Perna C, et al. UpRegulation of miR-34b/C by JNK and FOXO3 Protects From Liver Fibrosis Proc Natl Acad Sci USA (2021) 118(10):1-10. doi: 10.1073/pnas.2025242118

36. Lappas M, Lim R, Riley C, Menon R, Permezel M. Expression and Localisation of FoxO3 and FoxO4 in Human Placenta and Fetal Membranes. Placenta (2010) 31(12):1043-50. doi: 10.1016/j.placenta.2010.09.009

37. Zhao Y, Wang Y, Zhu WG. Applications of Post-Translational Modifications of FoxO Family Proteins in Biological Functions. J Mol Cell Biol (2011) 3 (5):276-82. doi: 10.1093/jmcb/mjr013

38. Galanos C, Freudenberg MA, Reutter W. Galactosamine-Induced Sensitization to the Lethal Effects of Endotoxin. Proc Natl Acad Sci USA (1979) 76(11):5939-43. doi: 10.1073/pnas.76.11.5939

39. Kato Y, Morikawa A, Sugiyama T, Koide N, Jiang GZ, Takahashi K, et al. Role of Tumor Necrosis Factor-Alpha and Glucocorticoid on Lipopolysaccharide (LPS)-Induced Apoptosis of Thymocytes. FEMS Immunol Med Microbiol (1995) 12(3-4):195-204. doi: 10.1111/j.1574-695X.1995.tb00192.x

40. Perlstein RS, Whitnall MH, Abrams JS, Mougey EH, Neta R. Synergistic Roles of Interleukin-6, Interleukin-1, and Tumor Necrosis Factor in the Adrenocorticotropin Response to Bacterial Lipopolysaccharide In Vivo. Endocrinol (1993) 132(3):946-52. doi: 10.1210/endo.132.3.8382602

41. Swain P, Nayak SK, Nanda PK, Dash S. Biological Effects of Bacterial Lipopolysaccharide (Endotoxin) in Fish: A Review. Fish Shellfish Immunol (2008) 25(3):191-201. doi: 10.1016/j.fsi.2008.04.009

42. Li P, Ye J, Zeng S, Yang C. Florfenicol Alleviated Lipopolysaccharide (LPS)Induced Inflammatory Responses in Ctenopharyngodon Idella Through Inhibiting Toll/NF-KappaB Signaling Pathways. Fish Shellfish Immunol (2019) 94:479-84. doi: 10.1016/j.fsi.2019.08.073

43. van Beek JH, de Moor MH, de Geus EJ, Lubke GH, Vink JM, Willemsen G, et al. The Genetic Architecture of Liver Enzyme Levels: GGT, ALT and AST. Behav Genet (2013) 43(4):329-39. doi: 10.1007/s10519-013-9593-y 
44. Ramesh M, Anitha S, Poopal RK, Shobana C. Evaluation of Acute and Sublethal Effects of Chloroquine (C18H26CIN3) on Certain Enzymological and Histopathological Biomarker Responses of a Freshwater Fish Cyprinus Carpio. Toxicol Rep (2018) 5:18-27. doi: 10.1016/j.toxrep.2017.11.006

45. Hossain MS, Koshio S, Ishikawa M, Yokoyama S, Sony NM, Dawood MAO, et al. Efficacy of Nucleotide Related Products on Growth, Blood Chemistry, Oxidative Stress and Growth Factor Gene Expression of Juvenile Red Sea Bream, Pagrus Major. Aquaculture (2016) 464:8-16. doi: 10.1016/j.aquaculture. 2016.06.004

46. Papa S, Bubici C, Zazzeroni F, Franzoso G. Mechanisms of Liver Disease: Cross-Talk Between the NF-KappaB and JNK Pathways. Biol Chem (2009) 390(10):965-76. doi: 10.1515/BC.2009.111

47. Essers MA, Weijzen S, de Vries-Smits AM, Saarloos I, de Ruiter ND, Bos JL, et al. FOXO Transcription Factor Activation by Oxidative Stress Mediated by the Small GTPase Ral and JNK. EMBO J (2004) 23(24):4802-12. doi: 10.1038/ sj.emboj.7600476

48. Sunayama J, Tsuruta F, Masuyama N, Gotoh Y. JNK Antagonizes AktMediated Survival Signals by Phosphorylating 14-3-3. J Cell Biol (2005) 170 (2):295-304. doi: 10.1083/jcb.200409117

49. Kong W, Li C, Qi Q, Shen J, Chang K. Cardamonin Induces G2/M Arrest and Apoptosis via Activation of the JNK-FOXO3a Pathway in Breast Cancer Cells. Cell Biol Int (2019) 44(1):177-88. doi: 10.1002/cbin.11217

50. Yang Y, Yu S, Liu N, Xu H, Gong Y, Wu Y, et al. Transcription Factor FOXO3a Is a Negative Regulator of Cytotoxicity of Fusarium Mycotoxin in GES-1 Cells. Toxicol Sci (2018) 166(2):370-81. doi: 10.1093/toxsci/kfy216

51. Lee HY, Youn SW, Kim JY, Park KW, Hwang CI, Park WY, et al. FOXO3a Turns the Tumor Necrosis Factor Receptor Signaling Towards Apoptosis Through Reciprocal Regulation of C-Jun N-Terminal Kinase and NF-KappaB. Arterioscler Thromb Vasc Biol (2008) 28(1):112-20. doi: 10.1161/ATVBAHA. 107.153304

52. Yu T, Ji J, Guo YL. MST1 Activation by Curcumin Mediates JNK Activation, Foxo3a Nuclear Translocation and Apoptosis in Melanoma Cells. Biochem Biophys Res Commun (2013) 441(1):53-8. doi: 10.1016/j.bbrc.2013.10.008
53. Li D, Li X, Wu J, Li J, Zhang L, Xiong T, et al. Involvement of the JNK/ FOXO3a/Bim Pathway in Neuronal Apoptosis After Hypoxic-Ischemic Brain Damage in Neonatal Rats. PLoS One (2015) 10(7):e0132998. doi: 10.1371/ journal.pone.0132998

54. Schwabe RF, Seki E, Brenner DA. Toll-Like Receptor Signaling in the Liver. Gastroenterology (2006) 130(6):1886-900. doi: 10.1053/j.gastro.2006.01.038

55. Berzsenyi MD, Roberts SK, Preiss S, Woollard DJ, Beard MR, Skinner NA, et al. Hepatic TLR2 \& TLR4 Expression Correlates With Hepatic Inflammation and TNF-Alpha in HCV \& HCV/HIV Infection. J Viral Hepat (2011) 18(12):852-60. doi: 10.1111/j.1365-2893.2010.01390.x

56. Jia R, Li Y, Cao L, Du J, Zheng T, Qian H, et al. Antioxidative, Anti-Inflammatory and Hepatoprotective Effects of Resveratrol on Oxidative Stress-Induced Liver Damage in Tilapia (Oreochromis Niloticus). Comp Biochem Physiol C Toxicol Pharmacol (2019) 215:56-66. doi: 10.1016/j.cbpc.2018.10.002

57. Ma JQ, Li Z, Xie WR, Liu CM, Liu SS. Quercetin Protects Mouse Liver Against $\mathrm{CCl}(4)$-Induced Inflammation by the TLR2/4 and MAPK/NF-KappaB Pathway. Int Immunopharmacol (2015) 28(1):531-9. doi: 10.1016/j.intimp. 2015.06 .036

58. Li S, Dong G, Moschidis A, Ortiz J, Benakanakere MR, Kinane DF, et al. P. Gingivalis Modulates Keratinocytes Through FOXO Transcription Factors. PLoS One (2013) 8(11):e78541. doi: 10.1371/journal.pone.0078541

Conflict of Interest: The authors declare that the research was conducted in the absence of any commercial or financial relationships that could be construed as a potential conflict of interest.

Copyright (c) 2021 Pan, Liu, Huang, Guo, Luo, Yang, Gao, Xu, Zhang and Mai. This is an open-access article distributed under the terms of the Creative Commons Attribution License (CC BY). The use, distribution or reproduction in other forums is permitted, provided the original author(s) and the copyright owner(s) are credited and that the original publication in this journal is cited, in accordance with accepted academic practice. No use, distribution or reproduction is permitted which does not comply with these terms. 